\title{
Oscillations Excited by Plasmoids Formed During Magnetic Reconnection in a Vertical Gravitationally Stratified Current Sheet
}

\author{
P. Jelínek ${ }^{1}(1)$, M. Karlický ${ }^{2}$, T. Van Doorsselaere ${ }^{3}$ (D) , and M. Bárta $^{2}$ \\ ${ }^{1}$ University of South Bohemia, Faculty of Science, Institute of Physics and Biophysics, Branišovská 1760, CZ-370 05 České Budějovice, \\ Czech Republic; pjelinek@prf.jcu.cz \\ ${ }^{2}$ Czech Academy of Sciences, v. v. i., Astronomical Institute, Fričova 258, CZ-251 65 Ondřejov, Czech Republic \\ ${ }^{3}$ Centre for mathematical Plasma Astrophysics (CmPA), Mathematics Department, KU Leuven, Celestijnenlaan 200B bus 2400, B-3001 Leuven, Belgium \\ Received 2016 December 9; revised 2017 August 17; accepted 2017 August 18; published 2017 September 26
}

\begin{abstract}
Using the FLASH code, which solves the full set of the 2D non-ideal (resistive) time-dependent magnetohydrodynamic (MHD) equations, we study processes during the magnetic reconnection in a vertical gravitationally stratified current sheet. We show that during these processes, which correspond to processes in solar flares, plasmoids are formed due to the tearing mode instability of the current sheet. These plasmoids move upward or downward along the vertical current sheet and some of them merge into larger plasmoids. We study the density and temperature structure of these plasmoids and their time evolution in detail. We found that during the merging of two plasmoids, the resulting larger plasmoid starts to oscillate with a period largely determined by $L / c_{\mathrm{A}}$, where $L$ is the size of the plasmoid and $c_{\mathrm{A}}$ is the Alfvén speed in the lateral parts of the plasmoid. In our model, $L / c_{\mathrm{A}}$ evaluates to $\sim 25 \mathrm{~s}$. Furthermore, the plasmoid moving downward merges with the underlying flare arcade, which causes oscillations of the arcade. In our model, the period of this arcade oscillation is $\sim 35 \mathrm{~s}$, which also corresponds to $L / c_{\mathrm{A}}$, but here $L$ means the length of the loop and $c_{\mathrm{A}}$ is the average Alfvén speed in the loop. We also show that the merging process of the plasmoid with the flare arcade is a complex process as presented by complex density and temperature structures of the oscillating arcade. Moreover, all these processes are associated with magnetoacoustic waves produced by the motion and merging of plasmoids.
\end{abstract}

Key words: magnetohydrodynamics (MHD) - methods: numerical - Sun: corona - Sun: flares - waves

Supporting material: animations

\section{Introduction}

Magnetohydrodynamic plasma waves and oscillations are omnipresent in the solar atmosphere and they play a very important role in many phenomena observed in solar and stellar atmospheres (Aschwanden 2005; Stepanov et al. 2012) as well as in the problem of the acceleration of the fast component of the solar wind (e.g., Li et al. 2004; Li \& Li 2008; Nakariakov et al. 2016).

The magnetically dominated solar plasma supports the propagation of various types of MHD waves (e.g., Nakariakov \& Verwichte 2005; De Moortel \& Nakariakov 2012; Yu et al. 2016). In solar flares, quasi-periodic pulsations (QPPs) are commonly observed in radio, soft X-ray, hard X-ray, ultraviolet, and even in gamma-ray emissions (Roberts et al. 1984; Aschwanden 1987; Fárník et al. 2003; Wang et al. 2005; Nakariakov et al. 2006, 2010, 2016).

The period of these oscillations ranges from sub-seconds to tens of minutes (Frost 1969; Parks \& Winckler 1969; Mészárosová et al. 2006; Tan 2008; Karlický et al. 2010b; Kupriyanova et al. 2010; Huang et al. 2014; Nisticò et al. 2014), and some of them were interpreted as manifestations of the transverse, kink mode (e.g., Kupriyanova et al. 2013), or sausage modes (e.g., Nakariakov et al. 2003), collectively supported by flare loops. When interpreted this way, the measured QPPs can be seismologically exploited to infer such key parameters as the magnetic field strength in the key region where flare energy is released (e.g., Chen et al. 2015, 2016; Guo et al. 2016). The damping time of QPPs is not yet very well measured, but it is around a few periods (e.g., Kolotkov et al. 2015). Several theoretical models have been proposed to explain the generation of these QPPs (Nakariakov \& Melnikov 2009; Van Doorsselaere et al. 2016). The formation of number of plasmoids in the context of QPPs is shown by Innocenti et al. (2015) in their kinetic particle-in-cell simulations.

Despite many theoretical models and the numerical results, it is not well understood how QPPS are generated, and the mechanisms are still debated in the literature. For a review of recent numerical results, see e.g., Pascoe (2014). Here, we study in detail the generation of transverse waves in a flare arcade impacted by plasmoids ejected from a current sheet. Our study deviates from Takasao et al. (2015) and Takasao \& Shibata (2016) in the sense that they consider a steady reconnection in a single X-point with the fixed resistivity which produces the outflow interacting with the flare arcade, whereas in our model we included effects of the tearing mode instability. Owing to this instability, plasmoids are formed in the whole current sheet. Then, these plasmoids not only mutually interact, but they are also carried in a bursty outflow from the main reconnection site downward where they interact with the flare arcade and cause oscillations of this arcade. Takasao \& Shibata (2016) focused on the oscillations excited by a quasi-steady reconnection outflow, neglecting the oscillations caused by plasmoids. Thus, our simulation is complementary to their simulations. An excitation of the fast magnetoacoustic waves by ejected plasmoids after their interaction with ambient magnetic field was also investigated by Yang et al. (2015). However, in contrast with our present work, they performed their calculations in the so-called interchange-reconnection scenario.

Apart from the fact that transverse waves excited by plasmoids may be the mechanism behind the observed QPPs in solar flares, 
they could also be important for the energy transport in solar flares. Fletcher \& Hudson (2008) point out that magnetic tension waves can play an important role in the transport of energy from the flare site to the lower atmospheric layers. The energy of the transverse waves is then transported down and dissipated in the chromosphere (Russell \& Fletcher 2013; Reep \& Russell 2016) where it can be used to accelerate energetic particles.

In our previous papers (Jelínek \& Karlický 2012; Jelínek et al. 2012, 2015b), we numerically investigated the behavior of fast magnetoacoustic waves in a Harris current sheet structure, but we did not consider resistivity. In the present paper, we included the resistivity to show not only the formation of plasmoids and their coalescence, similar to the paper by Bárta et al. (2008), but also the collision of plasmoids outflowing from the reconnection site with the flare magnetic arcade, triggering oscillatory processes.

In real flares, for a sufficiently fast reconnection process, anomalous resistivity needs to be considered. This resistivity is generated at locations where the drift of electrons, forming the electric current, overcomes some critical velocity (e.g., the ionsound or even electron thermal velocity) (Norman \& Smith 1978). In solar flare conditions it requires a very narrow current sheet $(10-100 \mathrm{~m})$. Thus, in any global MHD model of solar flares it is difficult to consider such a very narrow current sheet and simultaneously high values of the very localized anomalous resistivity. Due to this problem, in the present paper we made a compromise: we take the current sheet that is much broader than that in a real flare and the resistivity which is greater than the collisional resistivity, but lower than the anomalous one. To be in agreement with observed oscillations of the flare arcade and plasmoid (Veronig et al. 2006; Karlický \& Kliem 2010), we chose the resistivity 100 times greater than the collisional one. Although in several aspects the model can be improved, it presents the processes that are new and important and still not considered in connection with solar flares.

Tajima et al. (1987) studied the merger process of two plasmoids and found that during this process a larger plasmoid is formed and it oscillates with the period of about the Alfvén transit time, i.e., $P \approx L / c_{\mathrm{A}}$, where $L$ is the characteristic width of the plasmoid and $c_{\mathrm{A}}$ is the mean Alfvén speed in the plasmoid. Plasmoids in solar flares have been observed in soft X-rays and EUV (Ohyama \& Shibata 1998; Kołomański \& Karlický 2007; Savage et al. 2012; Takasao et al. 2012). In some cases, the processes connected with these plasmoids produce the so-called drifting pulsating structures (DPSs) in the decimetric range of radio waves (Kliem et al. 2000; Bárta et al. 2008, 2011; Karlický \& Bárta 2011).

This paper is structured as follows. In Section 2, we present our numerical model, including the initial equilibrium and perturbations. The results of the numerical simulations and their interpretation are summarized in Section 3. Finally, we present some conclusions in the last Section 4.

\section{Model}

\subsection{Governing Equations}

Our numerical model describes a gravitationally stratified solar atmosphere in which the plasma dynamics are described by the 2D, time-dependent non-ideal (resistive) MHD equations. We use the FLASH code (Lee \& Deane 2009; Lee 2013), where the MHD equations are formulated in conservative form as

$$
\begin{gathered}
\frac{\partial \varrho}{\partial t}+\nabla \cdot(\varrho \boldsymbol{v})=0, \\
\frac{\partial \varrho \boldsymbol{v}}{\partial t}+\nabla \cdot(\varrho \boldsymbol{v} \boldsymbol{v}-\boldsymbol{B B})+\nabla p_{*}=\varrho \boldsymbol{g}, \\
\frac{\partial \varrho E}{\partial t}+\nabla \cdot\left[\left(\varrho E+p_{*}\right) \boldsymbol{v}-\boldsymbol{B}(\boldsymbol{v} \cdot \boldsymbol{B})\right] \\
=\varrho \boldsymbol{g} \cdot \boldsymbol{v}+\nabla \cdot(\boldsymbol{B} \times(\eta \nabla \times \boldsymbol{B})), \\
\frac{\partial \boldsymbol{B}}{\partial t}+\nabla \cdot(\boldsymbol{v B}-\boldsymbol{B v})=-\nabla \times(\eta \nabla \times \boldsymbol{B}), \\
\nabla \cdot \boldsymbol{B}=0 .
\end{gathered}
$$

Here, $\varrho$ is the mass density, $\boldsymbol{v}$ is the flow velocity, $\boldsymbol{B}$ is the magnetic field strength, $\boldsymbol{g}=\left[0,-g_{\odot}, 0\right]$ is the gravitational acceleration with $g_{\odot}=274 \mathrm{~ms}^{-2}$, and $\eta$ is the magnetic diffusivity. According to Priest (1982), the magnetic diffusivity in the collisional regime can be expressed as $\eta=10^{9} T^{-3 / 2}$, which gives us for the temperature $T \approx 10^{6} \mathrm{~K}$ a value $1 \mathrm{~m}^{2} \mathrm{~s}^{-1}$. To see the magnetic reconnection in our models at reasonable times, we tested different values of the magnetic diffusivity and finally used the value that is 100 times higher than the collisional diffusivity, but much smaller than the anomalous one. Although we use the constant resistivity over the whole simulation box, effects of this resistivity are localized only in the regions with high current densities, i.e., in the whole current sheet. It is more realistic then using an enhanced resistivity in a fixed point.

The total pressure $p_{*}$ is given by

$$
p_{*}=\left(p+\frac{B^{2}}{2 \mu_{0}}\right),
$$

where $p$ is the fluid thermal pressure and $B$ is the magnitude of the magnetic field. The specific total energy $E$ in Equation (3) is expressed as

$$
E=\epsilon+\frac{v^{2}}{2}+\frac{B^{2}}{2 \mu_{0} \varrho},
$$

where $\epsilon$ is the specific internal energy,

$$
\epsilon=\frac{p}{(\gamma-1) \varrho}
$$

with the adiabatic coefficient $\gamma=5 / 3, v$ is the magnitude of the flow velocity, and $\mu_{0}=1.26 \times 10^{-6} \mathrm{Hm}^{-1}$ is the magnetic permeability of free space.

Generally, the terms expressing the radiative losses $R_{\text {loss }}$, thermal conduction $T_{\text {cond }}$, and heating $H$ should be added to the set of MHD equations. These terms are certainly important in building a realistic flare model. However, at this stage in so far as the oscillatory behavior associated with plasmoids is of primary interest, we neglect these terms. However, because of the lack of these terms, the specific values of the periods numerically found in this work can be taken very carefully.

\subsection{Initial Equilibrium}

For a static $(\boldsymbol{v}=0)$ equilibrium, the Lorentz and gravity forces must be balanced by the pressure gradient in the entire 


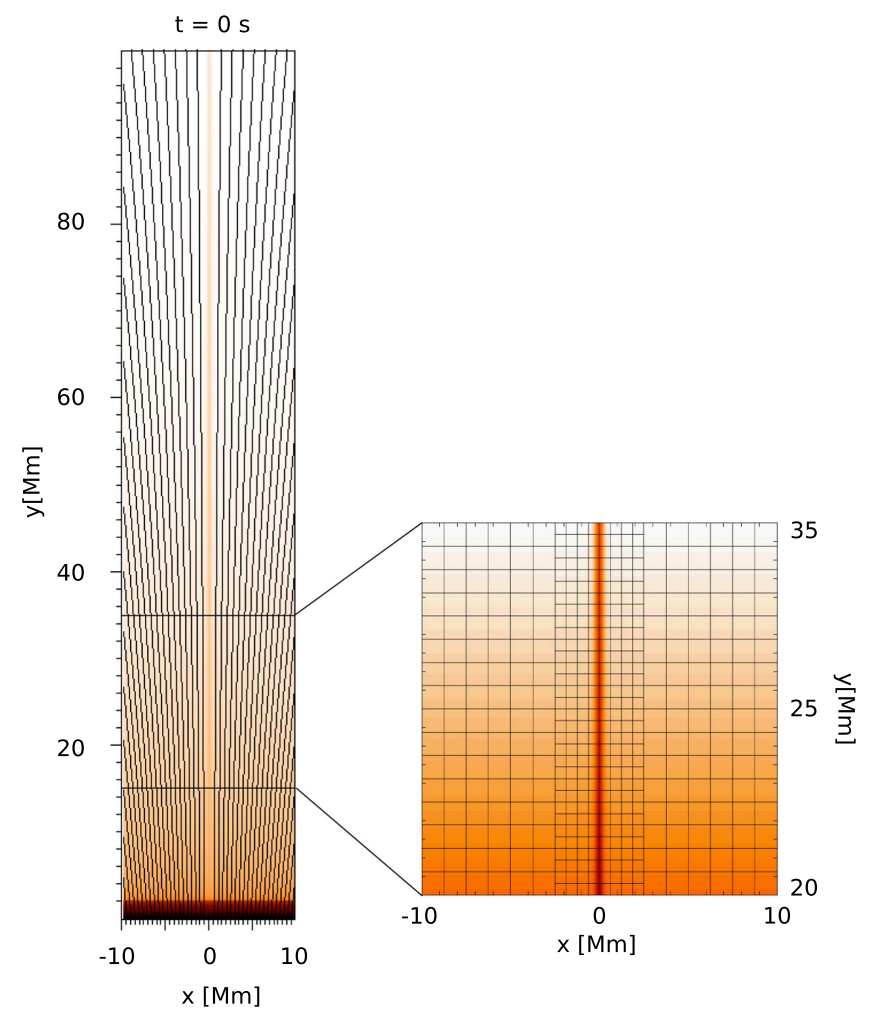

Figure 1. Left: initial mass density distribution over-plotted by the magnetic field structure as black lines. Right: the detail of the simulation region in the vicinity of perturbation point $(25 \mathrm{Mm})$, illustrating the computational grid, with AMR.

physical domain:

$$
-\nabla p+\boldsymbol{j} \times \boldsymbol{B}+\varrho \mathbf{g}=\boldsymbol{0} .
$$

The solenoidal condition, $\nabla \cdot \boldsymbol{B}=0$, is identically satisfied by using a magnetic flux function, $\boldsymbol{A}$,

$$
\boldsymbol{B}=\nabla \times \boldsymbol{A} .
$$

To calculate the magnetic field in the vertically oriented current sheet, we use the magnetic flux function $\boldsymbol{A}=\left[0,0, A_{z}\right]$ as e.g., Jelínek et al. (2012, 2015b):

$$
A_{z}=-B_{0} w_{\mathrm{cs}} \ln \left\{\left|\cosh \left(\frac{x}{w_{\mathrm{cs}}}\right)\right|\right\} \exp \left(-\frac{y}{\lambda}\right) .
$$

Here, the coefficient $\lambda \approx 100 \mathrm{Mm}$ denotes the magnetic scale height. The symbol $B_{0}$ is used for the external magnetic field and $w_{\mathrm{cs}}$ is the half width of the current sheet. We set $w_{\mathrm{cs}}=0.15 \mathrm{Mm}$. Note that the prescription for $\boldsymbol{g}$ and $\boldsymbol{A}$ implies a current sheet in the vertical direction $y$ with the current density flowing in the invariant direction $z ; x$ being the horizontal direction perpendicular to the current sheet.

The equilibrium gas pressure and mass density are computed according to the following equations; see e.g., Solov'ev (2010) and Jelínek et al. (2015a):

$$
p(x, y)=p_{\mathrm{h}}(y)-\frac{1}{\mu_{0}}\left[\int_{-\infty}^{x} \frac{\partial^{2} A}{\partial y^{2}} \frac{\partial A}{\partial x} d x+\frac{1}{2}\left(\frac{\partial A}{\partial x}\right)^{2}\right],
$$

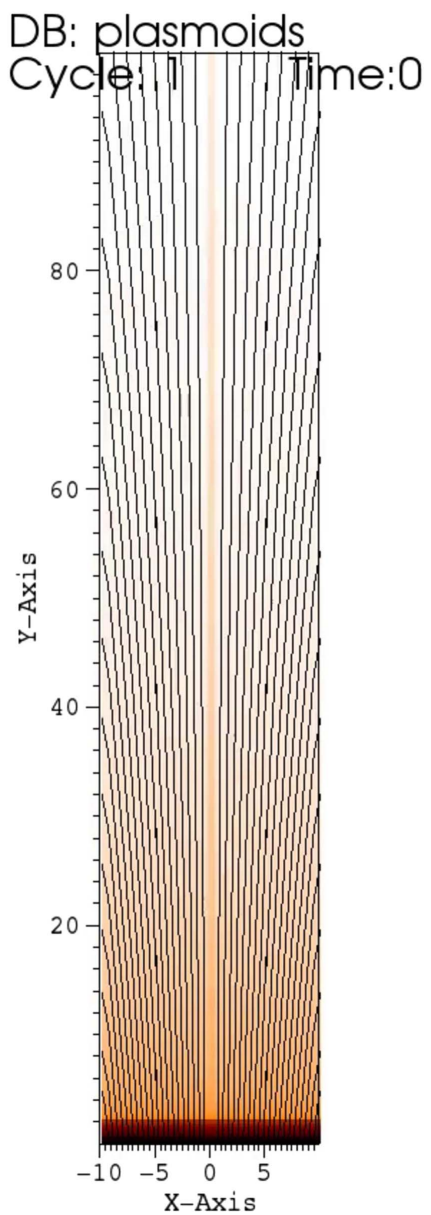

Figure 2. Numerical simulation showing the mass density and corresponding magnetic field lines evolving from $t=0$ to $250 \mathrm{~s}$.

(An animation of this figure is available.)

$$
\begin{aligned}
\varrho(x, y)= & \varrho_{\mathrm{h}}(y)+\frac{1}{\mu_{0} g_{\odot}}\left\{\frac { \partial } { \partial y } \left[\int_{-\infty}^{x} \frac{\partial^{2} A}{\partial y^{2}} \frac{\partial A}{\partial x} d x\right.\right. \\
& \left.\left.+\frac{1}{2}\left(\frac{\partial A}{\partial x}\right)^{2}\right]-\frac{\partial A}{\partial y} \nabla^{2} A\right\} .
\end{aligned}
$$

Through the use of Equation (11) in these general formulas, we obtain the expressions for the equilibrium gas pressure

$$
\begin{aligned}
p(x, y)= & p_{h}(y)-\frac{B_{0}^{2}}{2 \mu_{0}}\left\{\frac{w_{\mathrm{cs}}^{2}}{\lambda^{2}} \ln ^{2}\left[\cosh \left(\frac{x}{w_{\mathrm{cs}}}\right)\right]\right. \\
& \left.+\tanh ^{2}\left(\frac{x}{w_{\mathrm{cs}}}\right)\right\} \exp \left(-\frac{2 y}{\lambda}\right)
\end{aligned}
$$

and mass density

$$
\begin{aligned}
\varrho(x, y)= & \varrho_{h}(y)-\frac{B_{0}^{2}}{\mu_{0} g_{\odot} \lambda}\left\{\tanh ^{2}\left(\frac{x}{w_{\mathrm{cs}}}\right)\right. \\
& \left.-\ln \left[\cosh \left(\frac{x}{w_{\mathrm{cs}}}\right)\right] \operatorname{sech}^{2}\left(\frac{x}{w_{\mathrm{cs}}}\right)\right\} \exp \left(-\frac{2 y}{\lambda}\right),
\end{aligned}
$$



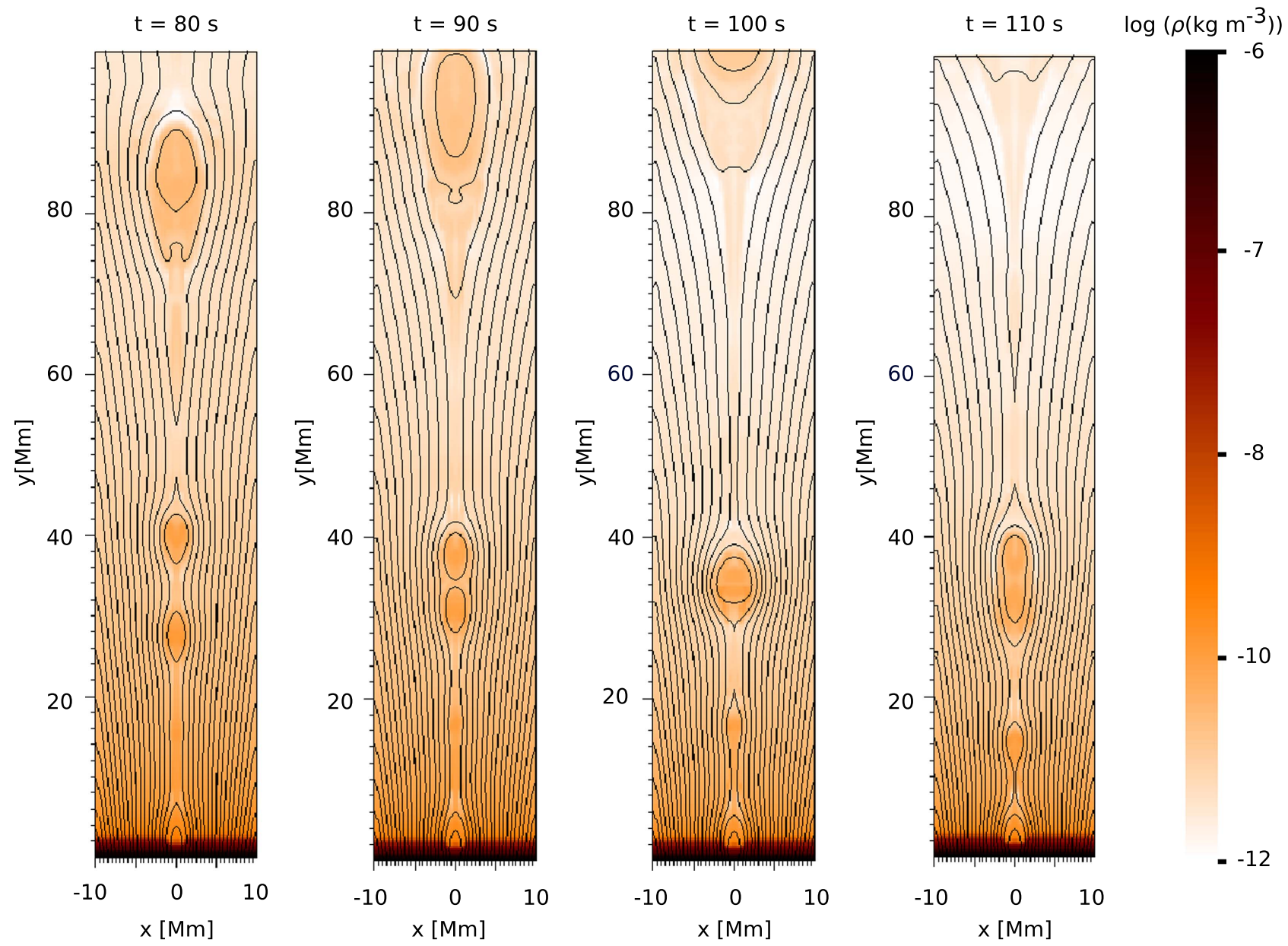

Figure 3. Mass density and the corresponding magnetic field lines, illustrating the merging of two plasmoids followed by an oscillation of the resulting plasmoid (see the processes in the $y=20-50 \mathrm{Mm}$ interval). The panels from left to right show times $t=80,90,100$, and $110 \mathrm{~s}$, respectively.

where

$$
p_{h}(y)=p_{0} \exp \left[-\int_{y_{0}}^{y} \frac{1}{\Lambda(\tilde{y})} d \tilde{y}\right]
$$

and

$$
\varrho_{h}(y)=\frac{p(y)}{g_{\odot} \Lambda(y)} .
$$

Here,

$$
\Lambda(y)=\frac{k_{\mathrm{B}} T(y)}{\bar{m} g_{\odot}}
$$

is the pressure scale height, which in the case of isothermal atmosphere represents the vertical distance over which the gas pressure decreases by a factor of $e \approx 2.7, k_{\mathrm{B}}=1.38 \times$ $10^{-23} \mathrm{~J} \mathrm{~K}^{-1}$ is the Boltzmann constant and $\bar{m}=0.6 m_{\mathrm{p}}$ is the mean particle mass $\left(m_{\mathrm{p}}=1.672 \times 10^{-27} \mathrm{~kg}\right.$ is the proton mass). $p_{0}$ in Equation (16) denotes the gas pressure at the reference level $y_{0}$. In our calculations, we set $y_{0}=10 \mathrm{Mm}$.

For the solar atmosphere, the temperature profile, $T(y)$, was derived by Vernazza et al. (1981), Avrett \& Loeser (2008). At the top of the photosphere, which corresponds to the height of $y=0.5 \mathrm{Mm}$, the temperature is $T(y)=5700 \mathrm{~K}$. At higher altitudes, the temperature falls down to its minimal value $T(y)=4350 \mathrm{~K}$ at $y \approx 0.95 \mathrm{Mm}$. Higher up, the temperature rises slowly to the height of about $y=2.7 \mathrm{Mm}$, where the transition region (TR) is located. Here, the temperature increases abruptly to the value $T(y)=1.5 \mathrm{MK}$, at the altitude $y=10 \mathrm{Mm}$, which is typical for the solar corona.

\subsection{Perturbations}

At the beginning of the numerical simulation $(t=0 \mathrm{~s})$, the equilibrium is perturbed by a Gaussian compression pulse in the $x$-component of velocity and has the following form:

$$
v_{x}=-A_{0} \frac{x}{\lambda_{x}} \exp \left[-\left(\frac{x}{\lambda_{x}}\right)^{2}\right] \exp \left[-\left(\frac{y-L_{\mathrm{P}}}{\lambda_{y}}\right)^{2}\right] \text {, }
$$

where $A_{0}=1 \mathrm{~km} \mathrm{~s}^{-1}$ is the initial amplitude of the pulse, and $\lambda_{x}=\lambda_{y}=0.15 \mathrm{Mm}$ are the widths of the velocity pulse in the longitudinal and transverse direction, respectively. Such a velocity pulse was originally used to generate sausage waves in coronal loops (Nakariakov et al. 2004, 2005). Here, we use this type of the velocity pulse to compress the magnetic field lines 


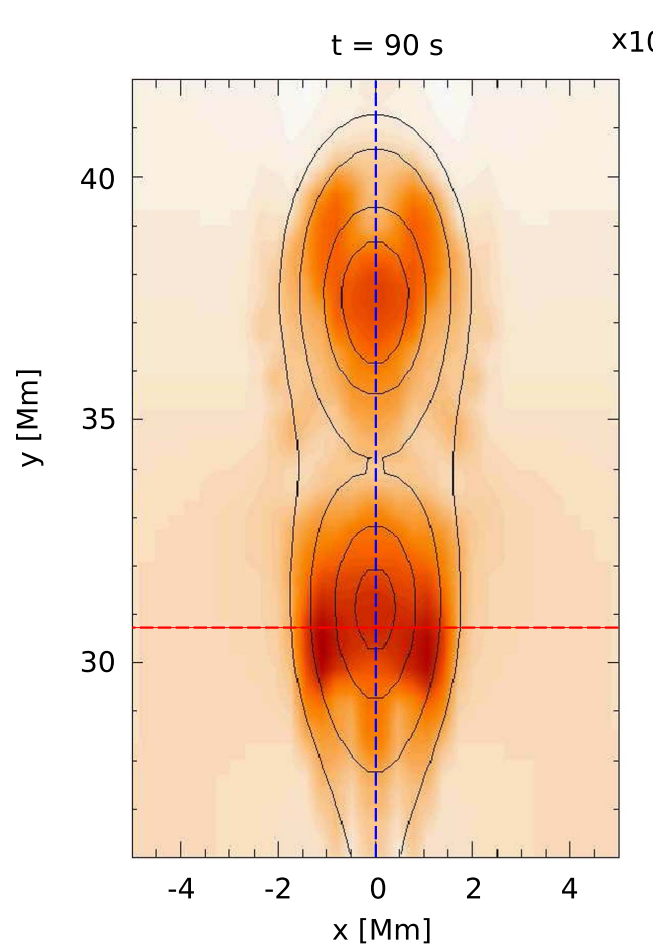

15

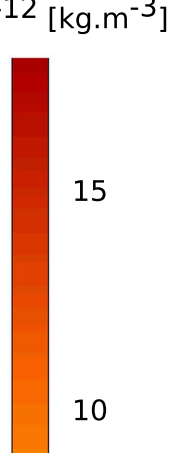

5

$\mathrm{x} 10^{-12}\left[\mathrm{~kg} \cdot \mathrm{m}^{-3}\right]$

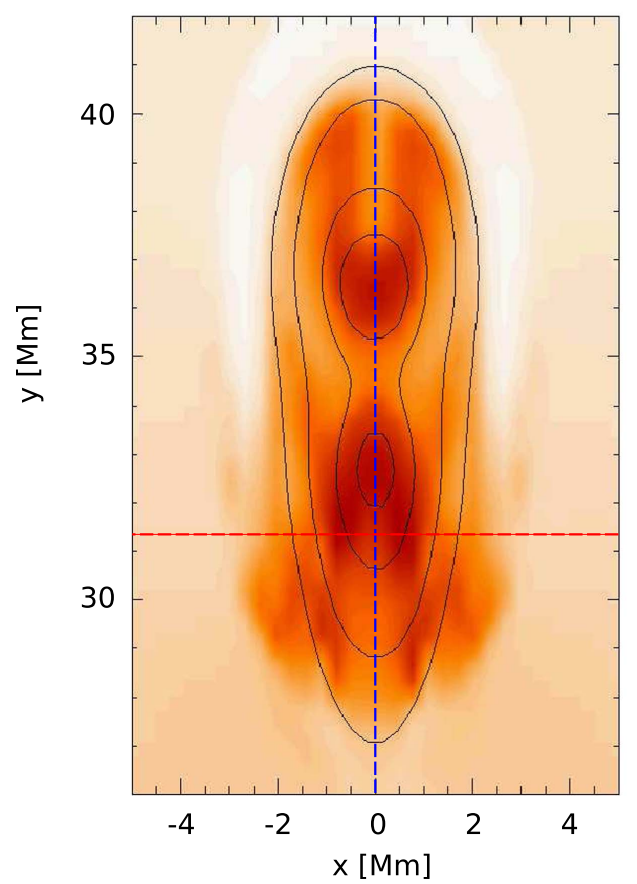

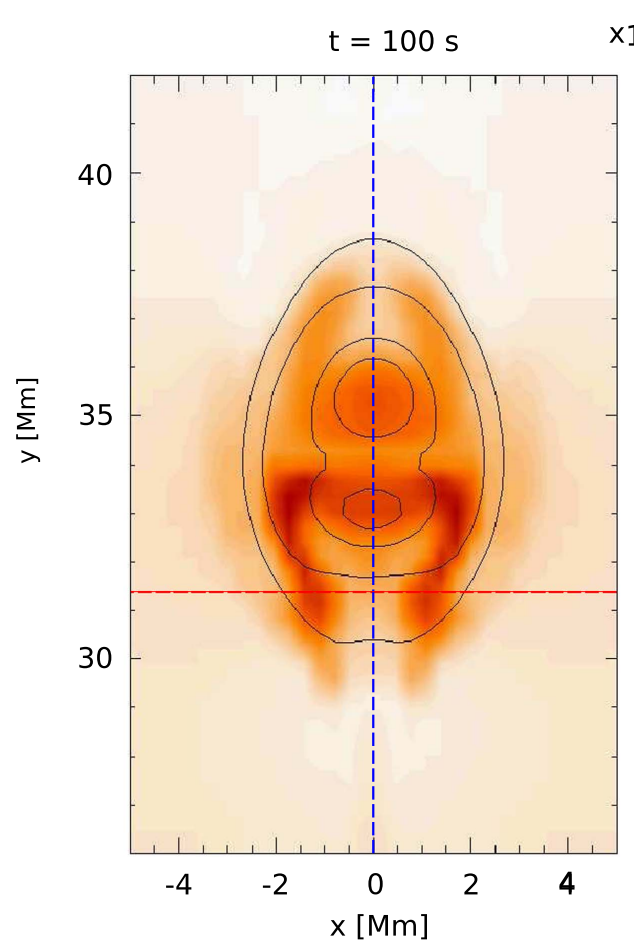

$\mathrm{x} 10^{-12}\left[\mathrm{~kg} \cdot \mathrm{m}^{-3}\right]$

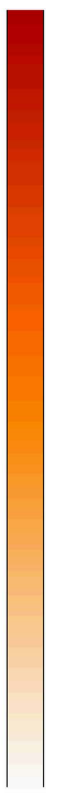

15

10

5

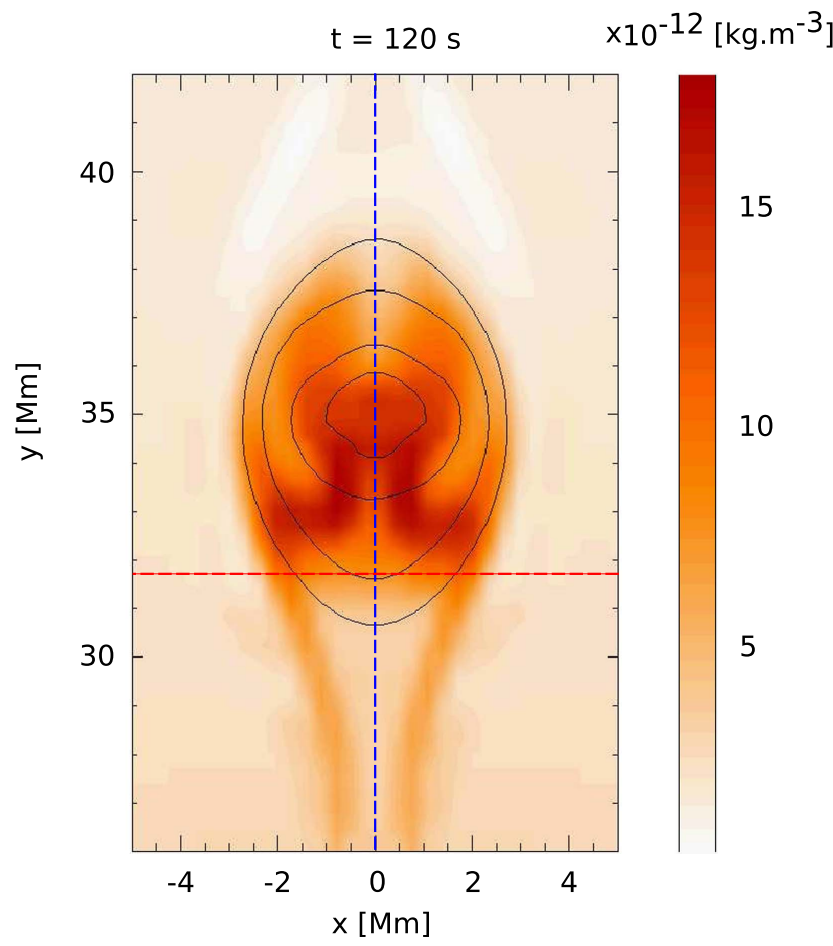

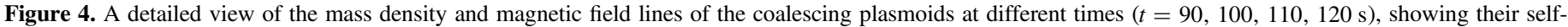
oscillations and complex density structure. An animation of the full simulation can be found in Figure 2 .

to initiate the magnetic reconnection in our numerical model. For such an initial velocity pulse, the ratio between the maximum of kinetic energy density and the background (thermal) energy density is $\approx 4.167 \times 10^{-5}$. The perturbation point $\left(x=0, y=L_{\mathrm{P}}\right)$ is located on the axis of the current sheet at a distance of $L=25 \mathrm{Mm}$ from the bottom boundary of the simulation region. This pulse is used to start the primary tearing instability in the current sheet. Later, because of the resistivity used in our model, a number of plasmoids as well as the magnetic arcade in the bottom part of the simulation region is formed self consistently.

\section{Numerical Solutions and Results}

We solve the 2D time-dependent, ideal MHD Equations (1)-(4) numerically through use of the FLASH code (Lee \& Deane 2009; Lee 2013). It is a well-tested, fully modular, parallel, multi-physics, 

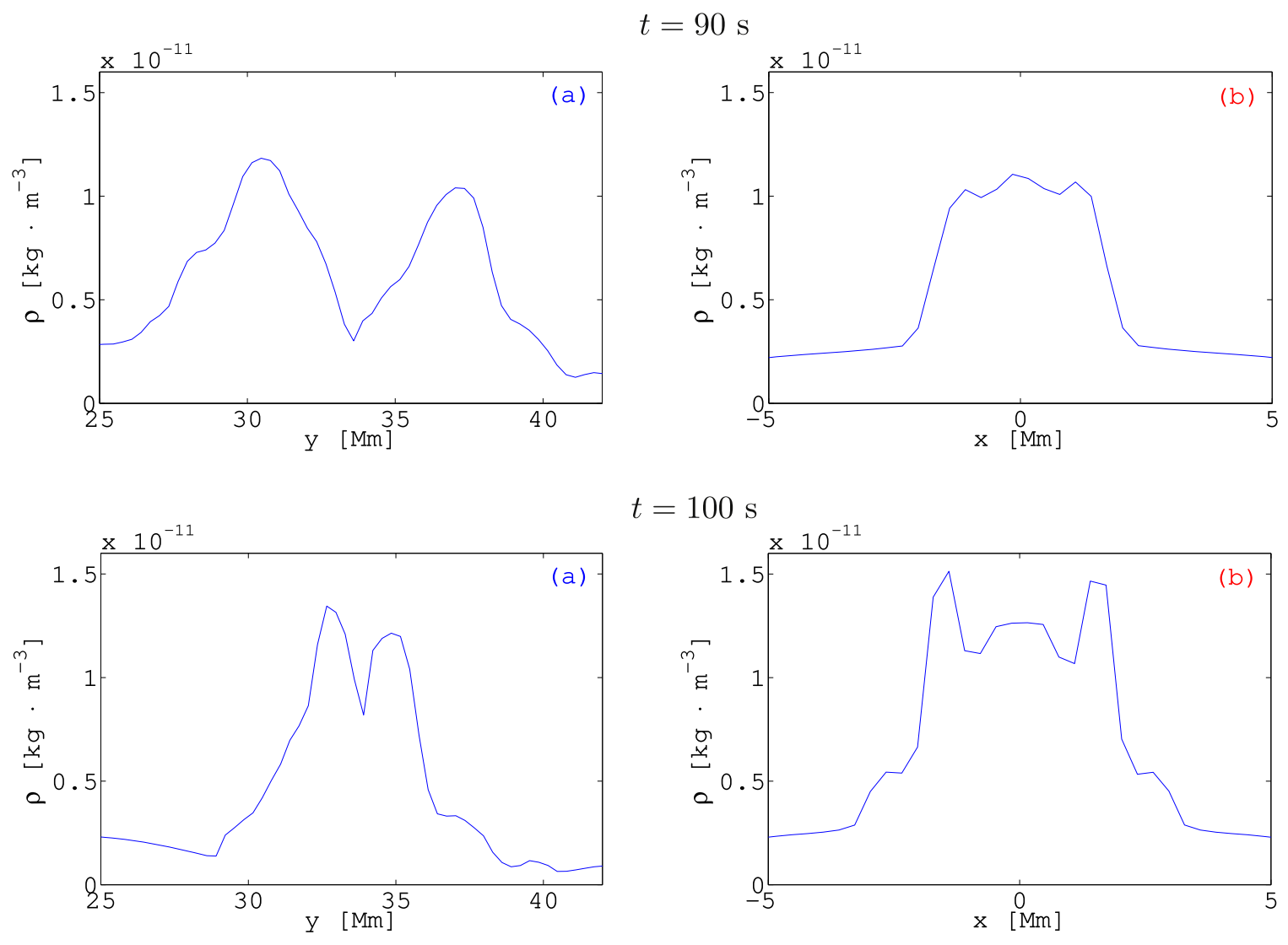

$t=100 \mathrm{~s}$
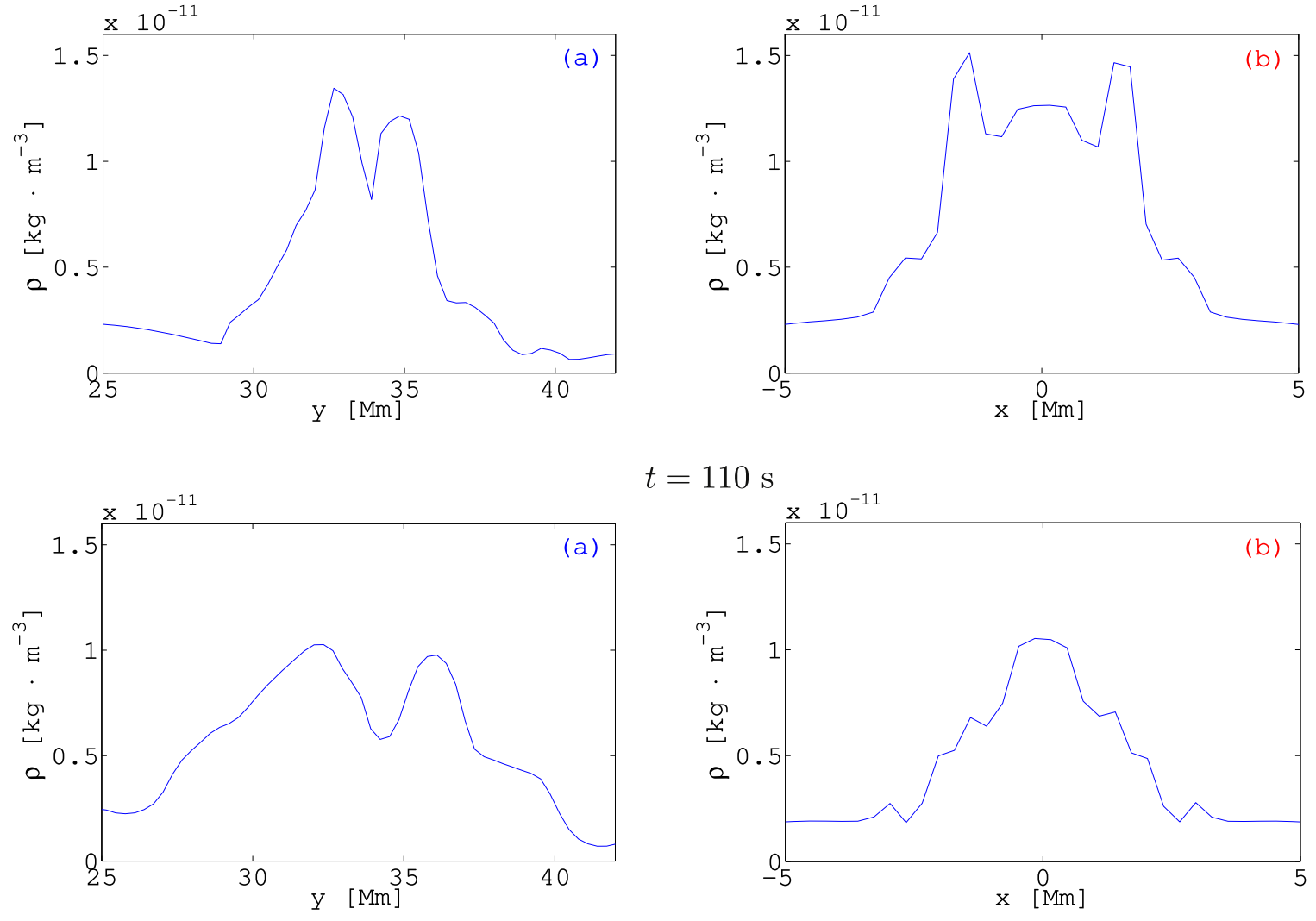

$t=110 \mathrm{~s}$
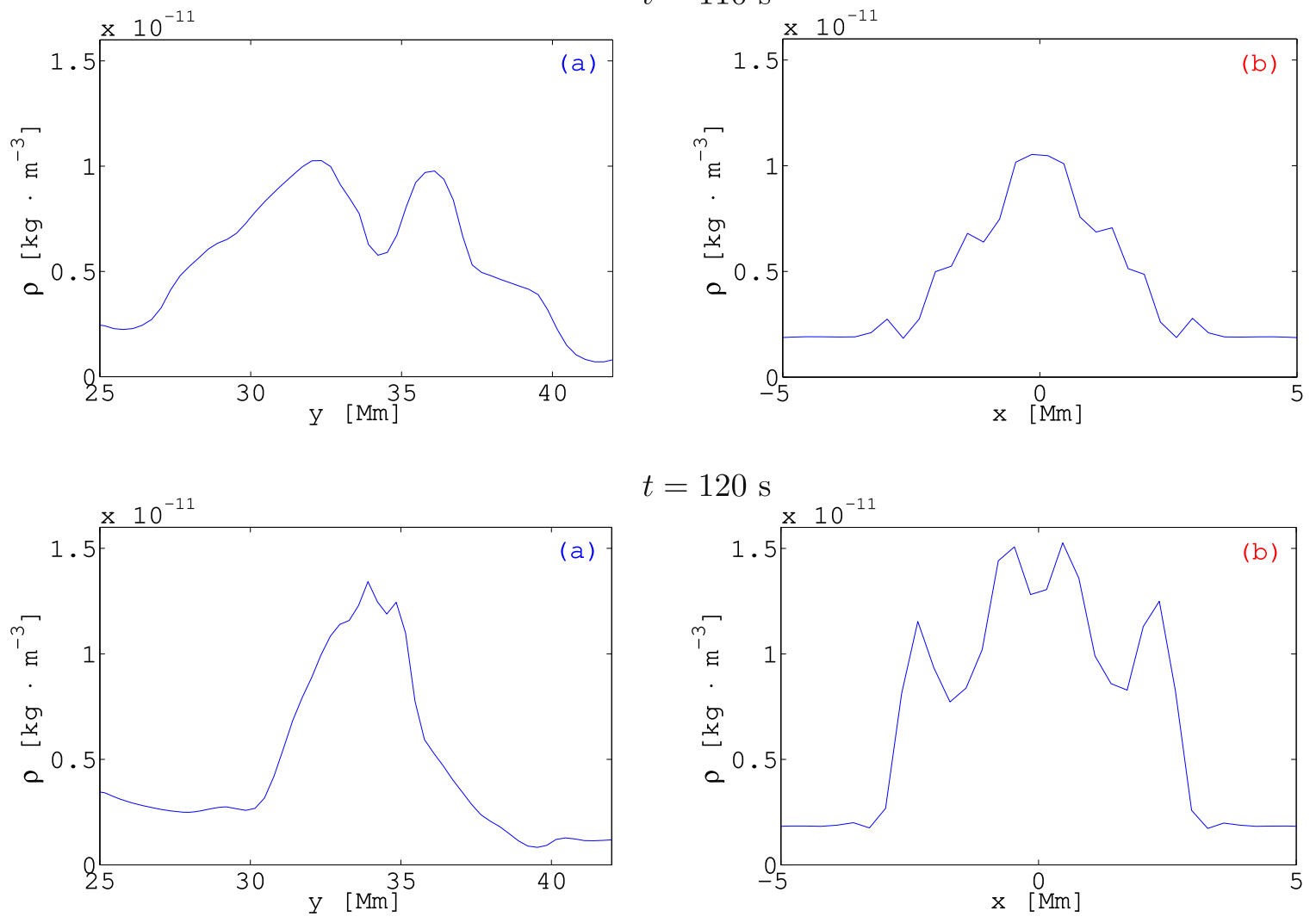

Figure 5. Density profiles along the vertical blue (a) and horizontal red (b) lines in Figure 4. 


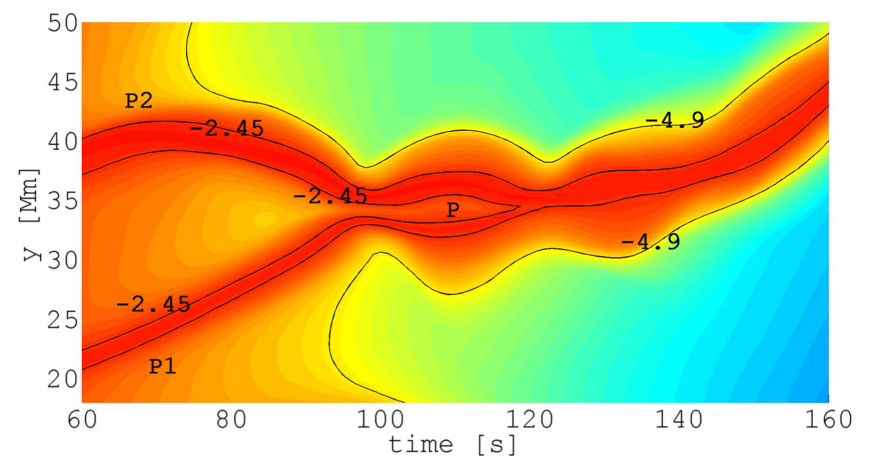

Figure 6. Temporal map of the vector potential at heights $18-50 \mathrm{Mm}$ above the solar surface. The solid black lines represent the positions of the magnetic field lines with the vector potential $A=-2.45$ and $A=-4.9$ along the axis of the current sheet during the merging of the two plasmoids (P1 and P2) into one plasmoid (P).

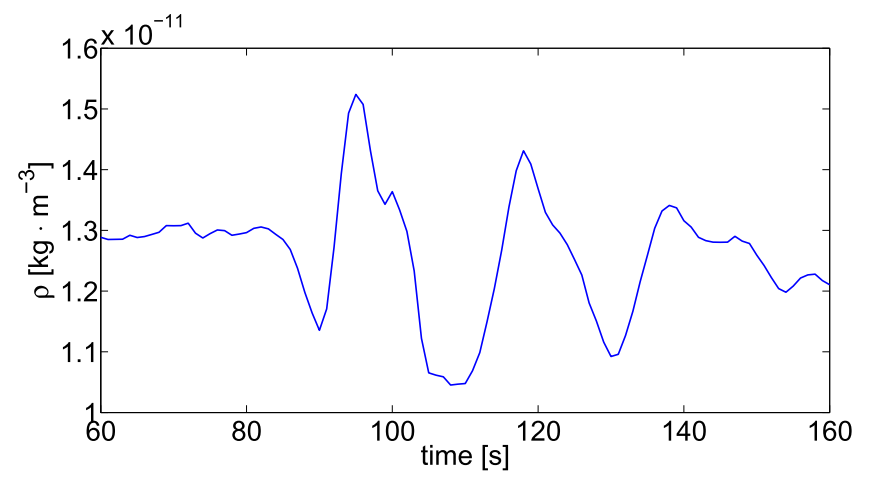

Figure 7. Evolution of the maximum mass density in the merging plasmoids, showing periodic behavior with a period $\approx 25 \mathrm{~s}$.

open science simulation code that implements second- and thirdorder unsplit Godunov solvers with various slope limiters and Riemann solvers, as well as adaptive mesh refinement (AMR, e.g., Chung 2002). The Godunov solver combines the corner transport upwind method for multi-dimensional integration and the constrained transport algorithm for preserving the divergence free constraint on the magnetic field (Lee \& Deane 2009). We use the minmod slope limiter and the Riemann solver in the Roe approximation (e.g., Toro 2006). The main advantage of using the AMR technique is to refine a numerical grid at steep spatial profiles while keeping the grid coarse at the places where fine spatial resolution is not essential. In our case, the AMR strategy is based on controlling the numerical errors near the gradient of mass density, leading to a reduction of the numerical diffusion within the entire simulation region.

For our numerical simulations, we use a 2D Eulerian box of height $H=100 \mathrm{Mm}$ and width $W=20 \mathrm{Mm}$. The spatial resolution of the numerical grid is determined with the AMR method and we use a similar setup as in the case of the vertical current sheet presented in Jelínek et al. (2015b). We use an AMR grid with the minimum (maximum) level of the refinement blocks set to 3(6) to have 203328 numerical cells (Figure 1). We use free boundary conditions, so that the incident waves could freely leave the simulation box without any significant reflection.

Prior to performing the numerical simulations, we verified that the system remains in numerical equilibrium for the

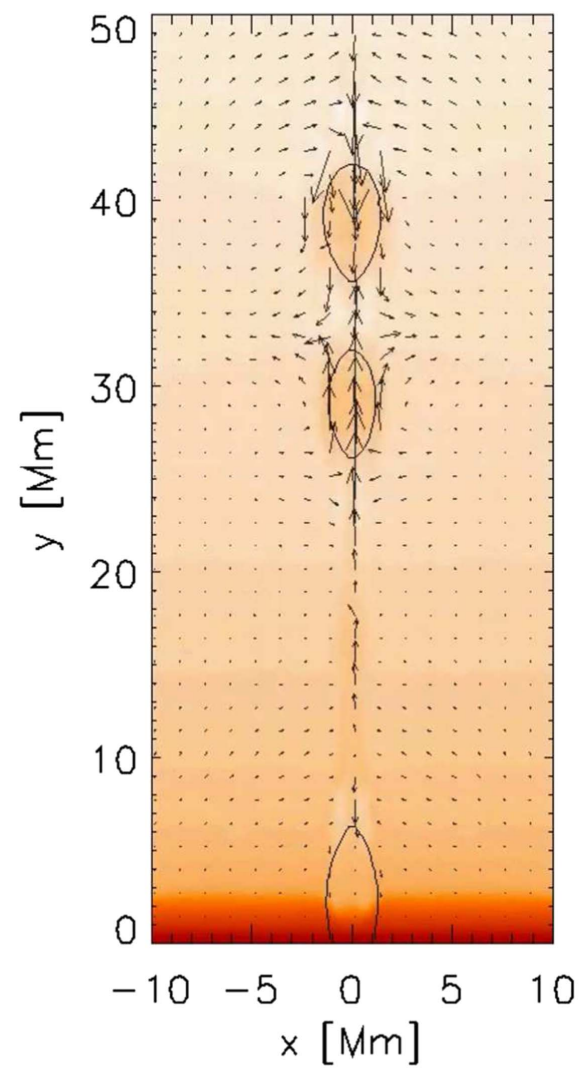

Figure 8. Animated plasma velocity field. The animation shows the plasma flows during and after the merging of plasmoids.

(An animation of this figure is available.)

adopted grid resolution by running a test simulation without any velocity pulse.

Very shortly after the initial pulse, the magnetic field reconnects and plasmoids (2D O-type magnetic field structures) are generated. The plasmoids move up and down in the current sheet, exciting oscillations in the plasmoids themselves and the magnetic arcade. These processes are described in the following subsections. For better visibility of oscillations and other processes, we also include an animation (Figure 2) from our numerical simulations.

\subsection{Plasmoid Merging and Oscillations}

In Figure 3, we show the mass density and magnetic field lines for times 80, 90, 100, and $110 \mathrm{~s}$, respectively. After the beginning of the magnetic reconnection, a number of plasmoids are formed in the vertical current sheet due to the tearing mode instability. During the simulation, some plasmoids merge into bigger ones and move upward through the corona and downward to the solar photosphere because of the gravitational or buoyancy forces. At time $t=80 \mathrm{~s}$ and in the $y=20-50 \mathrm{Mm}$ interval (Figure 3, left-most panel), there are two plasmoids that move toward each other. Around time $t=90 \mathrm{~s}$ (Figure 3, second panel), they start to merge into one larger plasmoid which then oscillates. These oscillations are clearly visible at times 100 and $110 \mathrm{~s}$. At that time, the oscillating plasmoid inverts its sense of propagation direction and starts to move up 


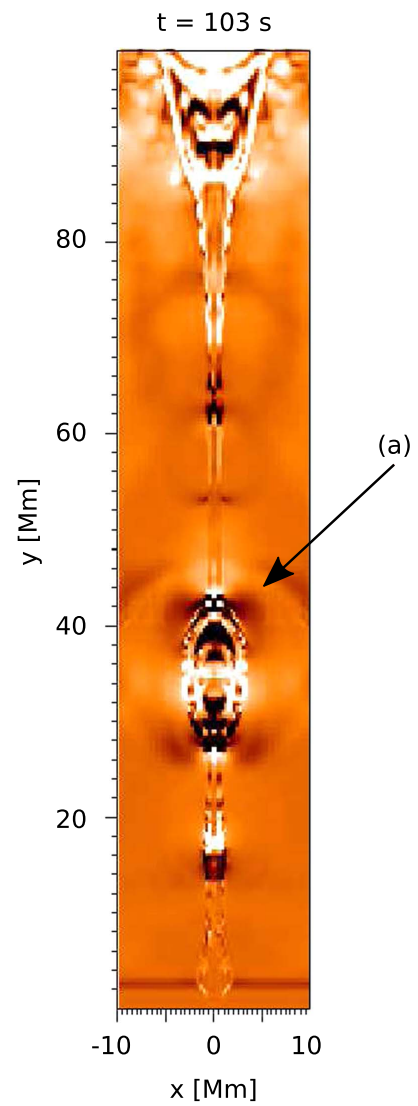

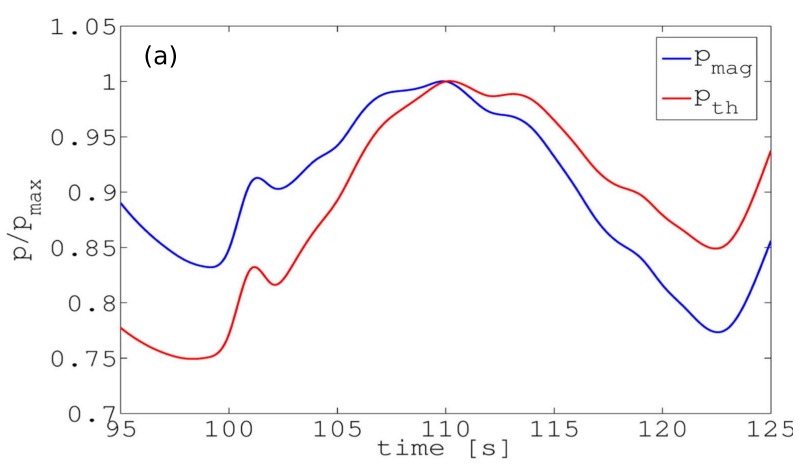

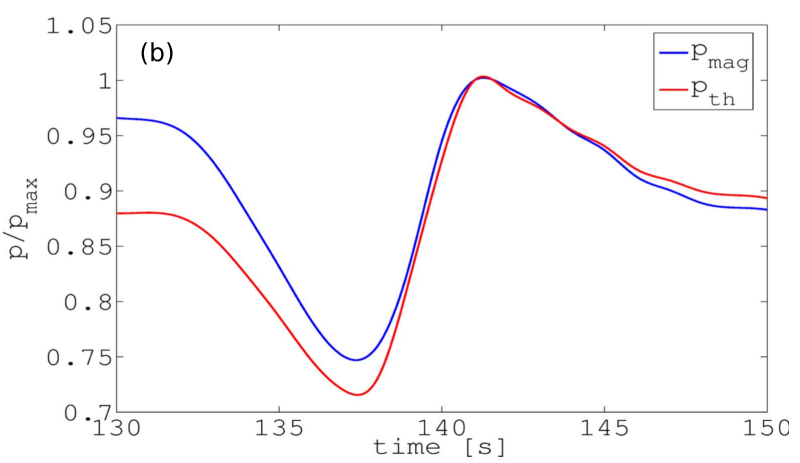

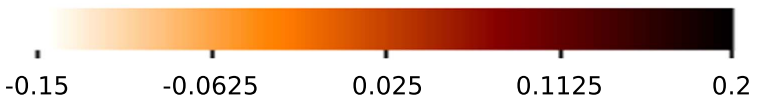

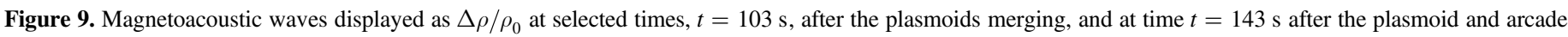

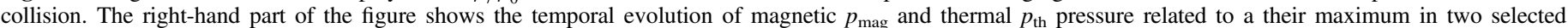

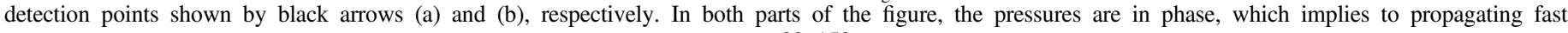
magnetoacoustic waves. The animation shows magnetoacoustic waves from $t=80-150 \mathrm{~s}$.

(An animation of this figure is available.)

(see the animated simulation of Figure 2). At the time around $160 \mathrm{~s}$, the oscillations are almost completely damped and the plasmoid slowly increases its size (in height $70-80 \mathrm{Mm}$ ) and at time around $200 \mathrm{~s}$ leaves freely the simulation region, as can also be seen in the animation.

Figure 4 provides a zoom-in for a detailed look at the behavior of the oscillating plasmoid and its inner structure for times 90, 100, 110 and $120 \mathrm{~s}$. The blue and red dashed lines correspond to a 1D density slice for both, a vertical direction and a horizontal direction, respectively. The positions of slices are chosen on the axis of symmetry of simulation region $(x=0.0 \mathrm{Mm})$, panels marked as (a), and at the place, where we found the maximum of mass density on this axis, panels marked as (b)- $(\approx 32-33 \mathrm{Mm})$, respectively; see Figure 5. Two peaks in graphs (a) for times 80-100 s represent two plasmoids that move toward each other and merge into one plasmoid which is finally represented by an increase of mass density in one peak visible at time $110 \mathrm{~s}$. In panels (b), we can recognize very nicely the complex internal plasmoid structure shortly before and after the merging process.

Figure 6 shows the interaction of the two plasmoids followed by oscillations of the merged larger plasmoid. Essentially, the figure shows the temporal map of the vector potential at heights between $18-50 \mathrm{Mm}$. To show clearly the plasmoids and their merging in this color map, we use only the selected values of the vector potential corresponding to their magnetic field lines. Here, we can see very clearly the plasmoid motion into higher altitudes of the solar atmosphere at times after $125 \mathrm{~s}$. The plasmoid $\left(\mathrm{P}_{1}\right)$ and plasmoid $\left(\mathrm{P}_{2}\right)$ are determined by two magnetic field lines (black solid lines) on the current sheet axis determined by the vector potentials $A=-2.45$ and $A=-4.9 . \mathrm{P}$ indicates the resulting plasmoid that starts to oscillate with a $\tau_{\text {plas }} \approx 25 \mathrm{~s}$ period after the merger from the two plasmoids $\left(\mathrm{P}_{1}\right)$ and $\left(\mathrm{P}_{2}\right)$. This period is very close to that calculated from the formula $\tau_{\text {plas }}=L / c_{\mathrm{A}}=27.9 \mathrm{~s}$, where $L \approx 6 \mathrm{Mm}$ is the size of plasmoid taken in our case from locations with the half of the maximal density in the plasmoid, and $c_{\mathrm{A}} \approx 0.215 \mathrm{Mm} \mathrm{s}^{-1}$ is the Alfvén speed calculated from the magnetic field and mass density in the same locations.

To show clearly the oscillatory process in the newly formed bigger plasmoid we present Figure 7. Here, we can see the temporal evolution of the maximum of the mass density on the axis of current sheet between the heights $25-42 \mathrm{Mm}$, where a clear oscillatory behavior of this plasmoid is observed. We can 

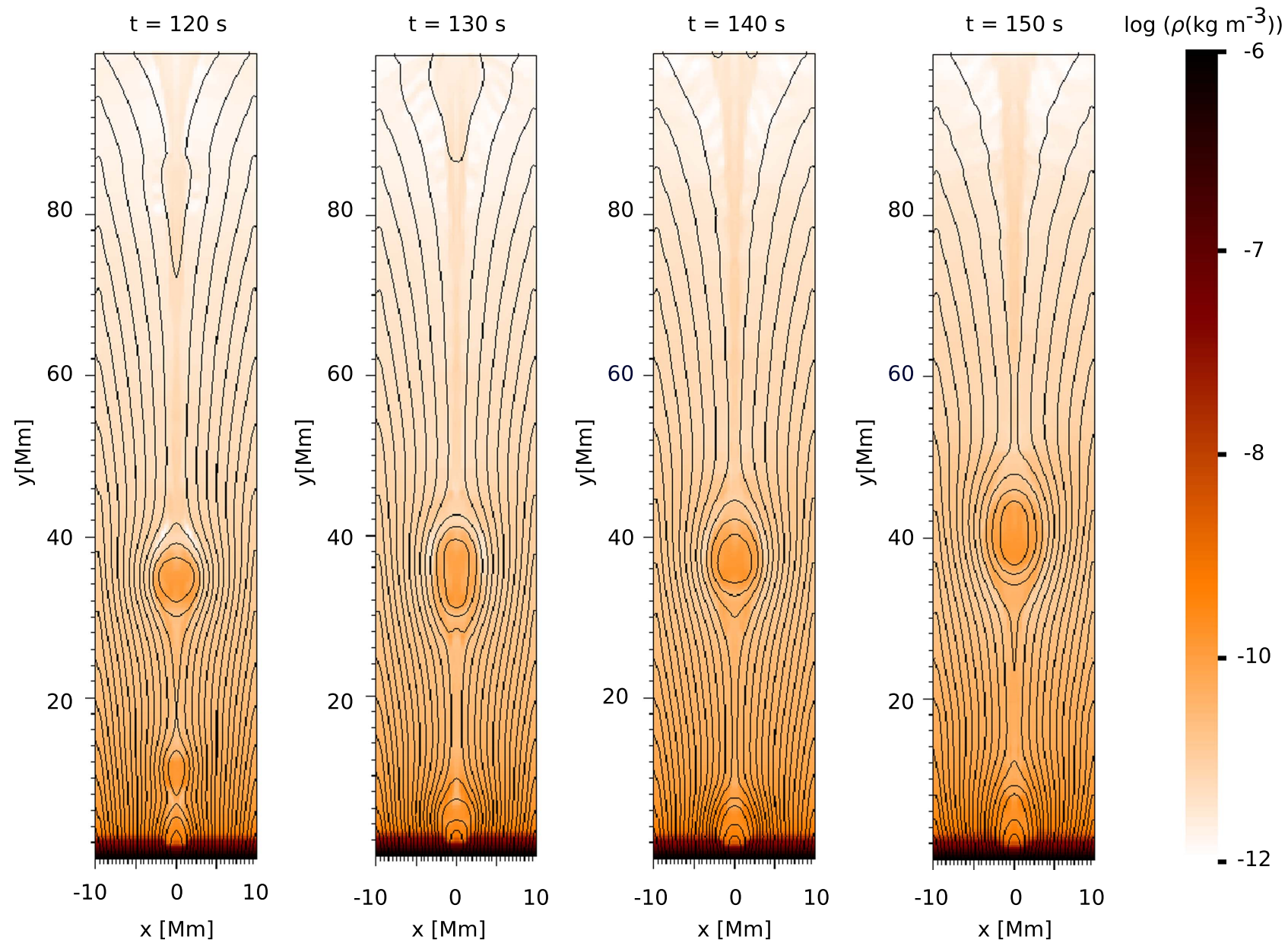

Figure 10. Mass density and corresponding magnetic field lines, illustrating the collision of the plasmoid with the magnetic arcade, showing its quickly damped oscillation (see the processes below $y=20 \mathrm{Mm}$ ). The panels from left to right show times $t=120,130,140$, and $150 \mathrm{~s}$, respectively. An animation of the full simulation can be found in Figure 2.

see here a wavy signal with an initial rarefaction around the time $\approx 90 \mathrm{~s}$ followed by a compression at time $\approx 95 \mathrm{~s}$. This initial phase continues with the next two oscillations which are very quickly attenuated, so that at time $\approx 160$ s they are damped entirely. First, we checked if this strong damping of oscillations is caused by the magnetic diffusivity. However, the diffusion time calculated as $\tau_{\text {damp }} \approx \mathcal{L}^{2} / \eta=2.5 \times 10^{9}$ s (see Roberts 1967), where $\mathcal{L} \approx 5 \mathrm{Mm}$ is the plasmoid width and the assumed magnetic diffusivity $\eta \approx 100 \mathrm{~m}^{2} \mathrm{~s}^{-1}$, is very long. Second, we performed a calculation with twice-higher spatial resolution and find that the damping time essentially was not changed. Furthermore, we made a special MHD test with a simple oscillating slab and we varied the resistivity in the interval from zero up to the resistivity, which is 100 times greater than the collisional resistivity (the resistivity used in this paper). In this test, in the mentioned interval of the resistivity, no change of the damping was found. Therefore, we made a detailed analysis and found that the plasmoid is not an isolated oscillating system, it is strongly coupled with plasma flows in its vicinity, and the damping (decrease of the oscillation amplitude) is caused by these mass plasma flows (see the Figure 8 animation) showing the plasma velocity field.

To complete our study, we present the magnetoacoustic waves (shown as $\Delta \rho / \rho_{0}$ ) in the simulation box; see the animation and snapshot in Figure 9. The wave generated by the plasmoid merging is clearly visible for the time $t=103 \mathrm{~s}$. The black arrow shows the detection point at $x=5 \mathrm{Mm}$ and $y=42 \mathrm{Mm}$, where we collect the variations of magnetic and thermal pressure, respectively. These time variations are depicted in the far right-hand side of figure labeled (a). Here, we can see that both pressures are in phase which means that we observe the fast magnetoacoustic wave (Aschwanden 2005).

\subsection{Arcade Oscillations}

Figure 10 shows the mass density and magnetic field lines for the times during the collision of the plasmoid with the magnetic arcade. At time $t=120 \mathrm{~s}$ and for $y$ below $20 \mathrm{Mm}$ (Figure 10, right-most panel), the plasmoid moves downwards to the magnetic arcade because of the gravitational force and tension of the surrounding magnetic field lines. Shortly after, at time $t=130 \mathrm{~s}$ (Figure 10, second panel), the plasmoid and arcade merge into one bigger arcade which starts to oscillate, as can be seen at times $t=140$ and $150 \mathrm{~s}$ (Figure 10, third and left-most panel; see also the animated simulation in Figure 2). From our numerical simulations, we found that the arcade oscillations are also very quickly damped. The reason for this 

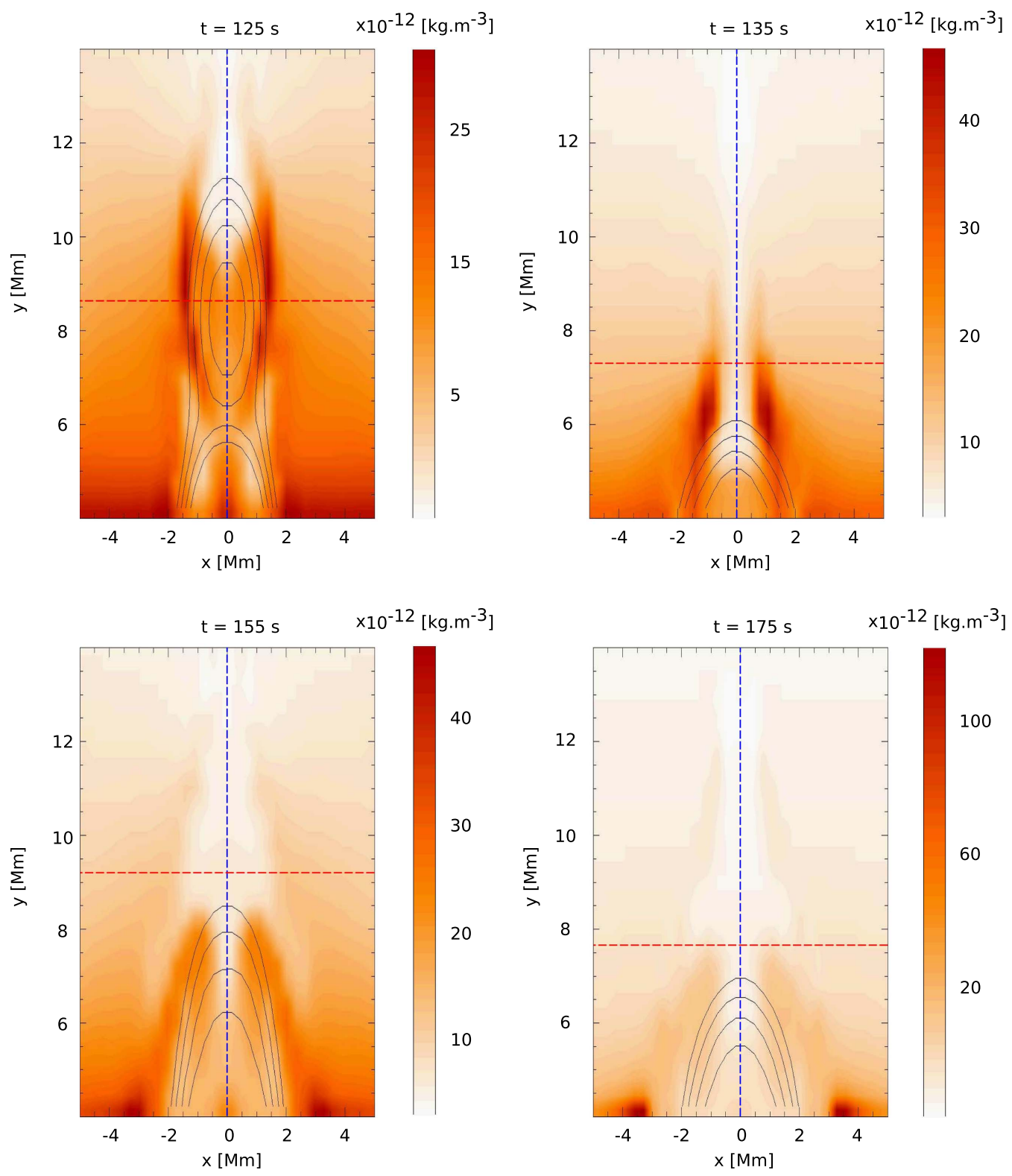

Figure 11. Detailed view of densities and magnetic field lines during the merging of the plasmoid with the arcade at times $t=125,135,155,175 \mathrm{~s}$.

damping is exactly the same as in the case of plasmoids oscillations. The arcade is not an isolated system; it is strongly coupled with processes in the current sheet. The downward oriented plasma flow, carrying plasmoids from reconnection toward the arcade, not only triggers the arcade oscillation, but in the following times the mass plasma flow damps this arcade oscillation (see also the animated simulation in Figure 2).

Figure 11 shows in detail the densities during the coalescence process, again together with the vertical (blue dashed line) and horizontal (red dashed line) slices. In Figure 12, we present the slices, which are, similarly as in Figure 5, in the positions of the axis of symmetry $(x=0.0 \mathrm{Mm})$ and the position where we find the maximum of mass density on this axis, i.e., $y \approx 7-9 \mathrm{Mm}$. In panel (a) for time $125 \mathrm{~s}$, the hump in mass density represents the plasmoid moving downwards to the magnetic arcade (see also the animated simulation in Figure 2). At time $135 \mathrm{~s}$, we observe the newly formed arcade that starts to oscillate; see the moving humps in panels (a) for times 155 and $175 \mathrm{~s}$ (compare with the temperature in Figure 17). Contrary to panels (b) displayed in Figure 5, the mass density in the center of the current sheet is lower than in the surrounding plasma. This is caused by the fact that the plasmoid is more dense than the plasma in the place where it was formed. However, because of the gravitational, downward acceleration of the plasma jet, the plasmoid moves down to lower altitudes of the solar atmosphere (TR), where the density is higher, thus reversing the density contrast.

In Figures 13 and 14, we present again the temporal maps of the vector potential at heights $2-18 \mathrm{Mm}$ above the solar surface. Similar to Figure 6, we draw selected vector potentials (magnetic field lines), showing clearly the oscillations of the top of the arcade.

Figure 13 shows a descent of the arcade top (determined by the magnetic field line in the current sheet axis with the vector 


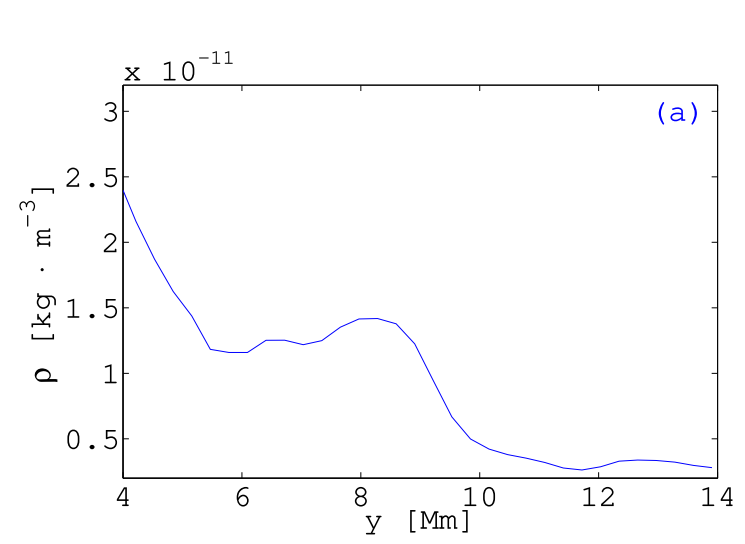

$t=125 \mathrm{~s}$
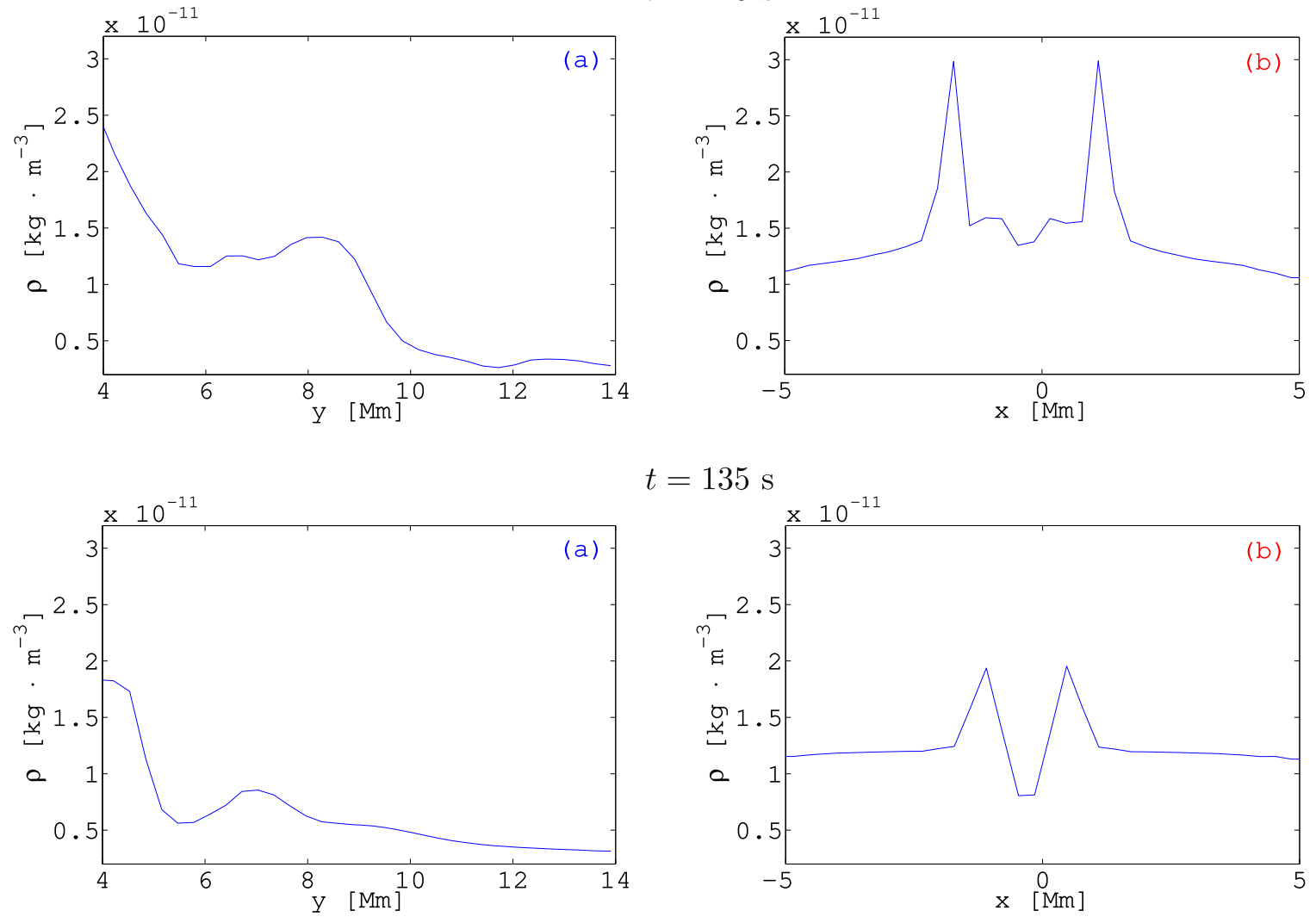

$t=135 \mathrm{~s}$

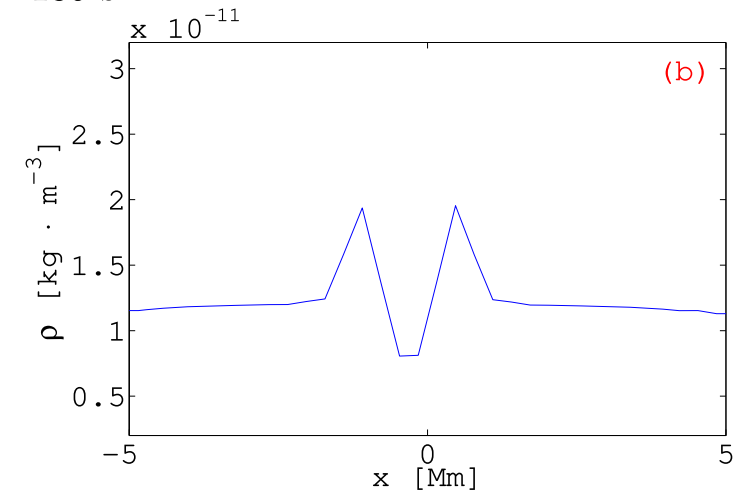

$t=155 \mathrm{~s}$
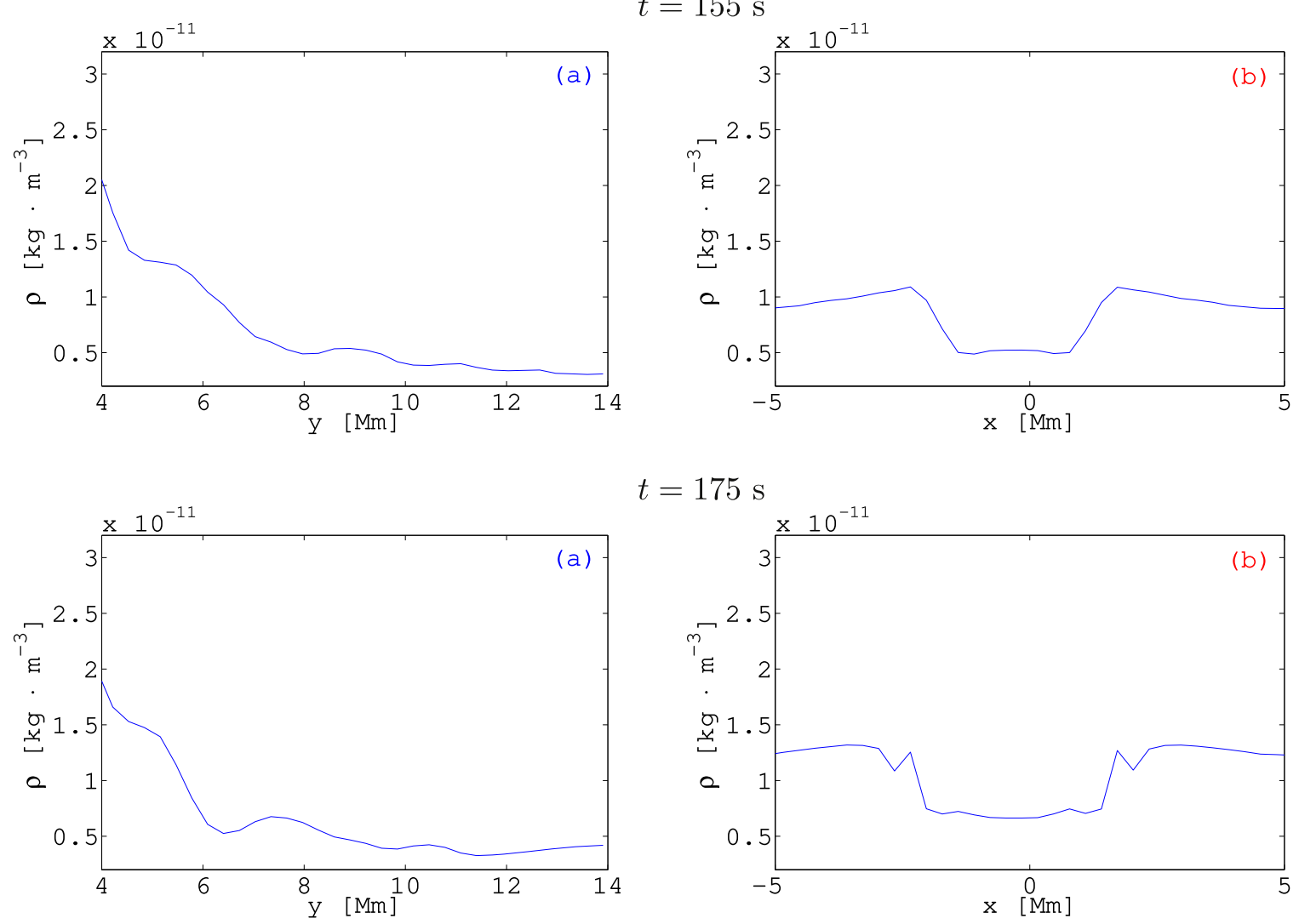

$t=175 \mathrm{~s}$

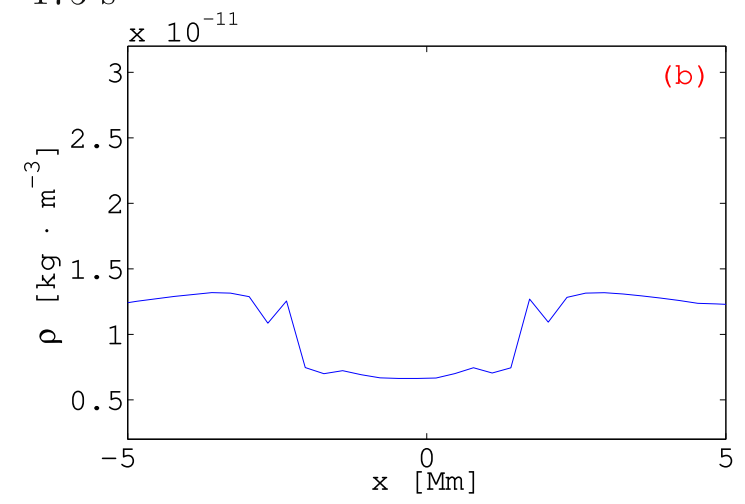

Figure 12. Density profiles along the vertical blue (a) and horizontal red (b) lines in Figure 11. 


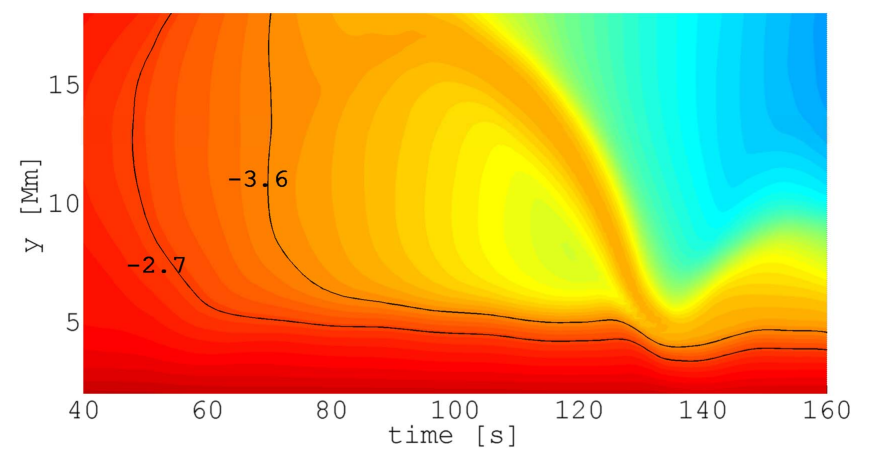

Figure 13. Temporal map of the vector potential at heights $2-18 \mathrm{Mm}$ above the solar surface. Here, we present the evolution of the arcade magnetic field lines having the vector potential $A=-2.7$ and $A=-3.6$, showing its decrease in height in the early phases.

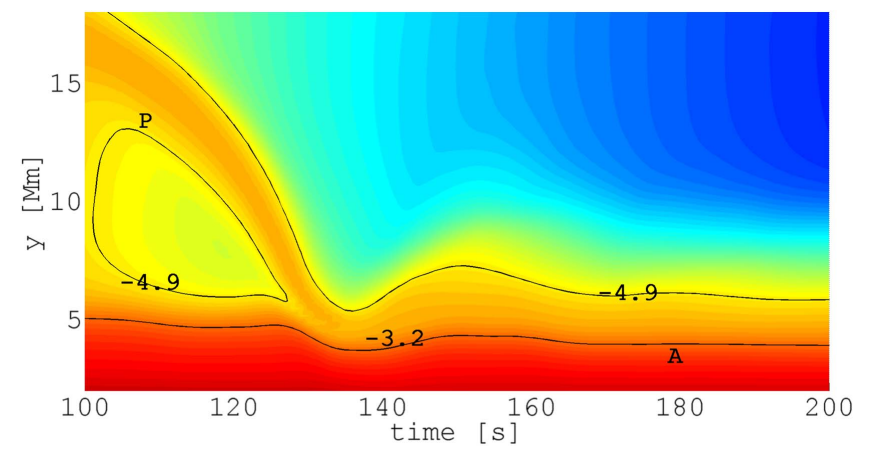

Figure 14. Temporal map of the vector potential at heights $2-18 \mathrm{Mm}$ above the solar surface. Here, we present the evolution of the height of the magnetic field lines at the top of the arcade, corresponding to the vector potential $A=-3.2$ and $A=-4.9$, showing the oscillation of the arcade (A) after its merging with the plasmoid $(\mathrm{P})$.

potential $A=-2.7$ and $A=-3.6)$ at the early stage of the magnetic field reconnection $(50-80 \mathrm{~s})$ in the vertical and gravitationally stratified current sheet. This process was already proposed to explain the downward motion of the X-ray source at the beginning of the 2003 November 3 flare (Veronig et al. 2006).

Figure 14 shows the interaction of the plasmoid with the arcade and clearly shows the subsequent arcade oscillations. The plasmoid (P) and arcade (A) are determined by two magnetic field lines at the current sheet axis with the vector potential $A=-3.2$ and $A=-4.9$. As seen here, after the interaction of the plasmoid with the arcade, the arcade starts to oscillate with a $35 \mathrm{~s}$ period. Moreover, it can be seen that oscillations of different layers of the arcade are not fully synchronized, as is evidenced by the phase lag between the maxima of the arcade oscillations expressed by the lines with $A=-3.2$ and $A=-4.9$.

Figure 15 presents the temporal evolution of the $y$ component of the velocity $-v_{y}$ in seven detection points along the selected magnetic field line with the vector potential $A=-3.8$. The left-hand panel of Figure 15 shows the signals from the left side of the arcade and on the right-hand panel shows signals from the right side of the arcade. The colors correspond to the positions (depending on the side of symmetry: left or right) as follows: black $(0.0 \mathrm{Mm})$, blue
(-0.3125 Mm; 0.3125 Mm), green (-0.625 Mm; 0.625 Mm) and red $(-0.9375 \mathrm{Mm} ; 0.9375 \mathrm{Mm})$, respectively. As can be seen here, the oscillations are synchronized at both sides of the arcade and quickly damped. Here, similar to the case of the damping of the oscillating plasmoid, we think that this damping is caused by a strong coupling between the oscillating flare arcade and plasma outflows from the above located magnetic reconnection. We estimate the period of the wave signal as $\tau_{\text {arc }} \approx 35 \mathrm{~s}$. Here as well, the estimated wave period corresponds to the formula $\tau_{\text {arc }}=L / c_{\mathrm{A}}=38.9 \mathrm{~s}$, where $L$ is a length of the loop and $c_{\mathrm{A}}$ is average Alfvén speed in the loop. A very important finding from our numerical simulation of the collision of a plasmoid and a magnetic arcade is that the impinging plasmoid can excite a transverse wave in the arcade as seen from both parts of Figure 15. We can infer that standing waves are observed in our simulations because the peaks of the wave signal are synchronized very well between symmetric locations, as seen in Figure 15. These standing, transverse waves may be responsible for the generation of the observed QPPs in solar flares.

To recognize the type of this standing wave, we expressed the temporal evolution of magnetic and thermal pressures in the arcade, in our case at position $x=+0.9375 \mathrm{Mm}$ and $y=4.1 \mathrm{Mm}$ (Figure 16). Both pressures are in anti-phase, showing that the standing wave is the slow magnetoacoustic wave (Aschwanden 2005).

As in the previous case, close to the arcade, which interacts with the plasmoid, we found the magnetoacoustic waves (see the animation and snapshot in Figure 9). We present the wave at time, $t=143 \mathrm{~s}$, in the position $x=5 \mathrm{Mm}$ and $y=6 \mathrm{Mm}$, marked by the arrow (b). The time variations of pressures are shown in the right-hand part of the figure. Both pressures are in the phase, which evidences again the fast magnetoacoustic wave.

To complete this part of our study, we show the time evolution of the temperature for different times 125, 135, 155, and $175 \mathrm{~s}$ in Figure 17 . We shifted the bottom boundary to higher altitudes of the solar atmosphere $(4 \mathrm{Mm})$ because of better readability in a linear temperature scale. We can clearly see that the plasmoid coalescence causes a temperature enhancement up to $\approx 80 \mathrm{MK}$. Note that in real flares, especially in high-temperature regions, there are deviations from our model where radiative and conduction losses are neglected. Thus, we expect that the real temperature will be lower. This temperature blob moves slowly down and after the collision and merging of the plasmoid with the arcade this blob starts to oscillate following the position of the top of the newly created magnetic arcade. During the observed oscillations, there is practically no change of the maximum of the temperature, so it remains almost at the same value.

The high temperature near the loop top may result in associated loop-top sources of flares. Therefore, the current mechanism of a merging plasmoid with a magnetic arcade could compete with, operate simultaneously, or help the previously proposed mechanism of Fang et al. (2016), who proposed that the inverse Compton scattering together with Kelvin-Helmholtz turbulence could lead to long-lived loop-top sources.

As for the energetic aspects of the merging, the kinetic, potential and magnetic energy of the plasmoid is completely absorbed in the arcade because there is no reflected plasmoid 

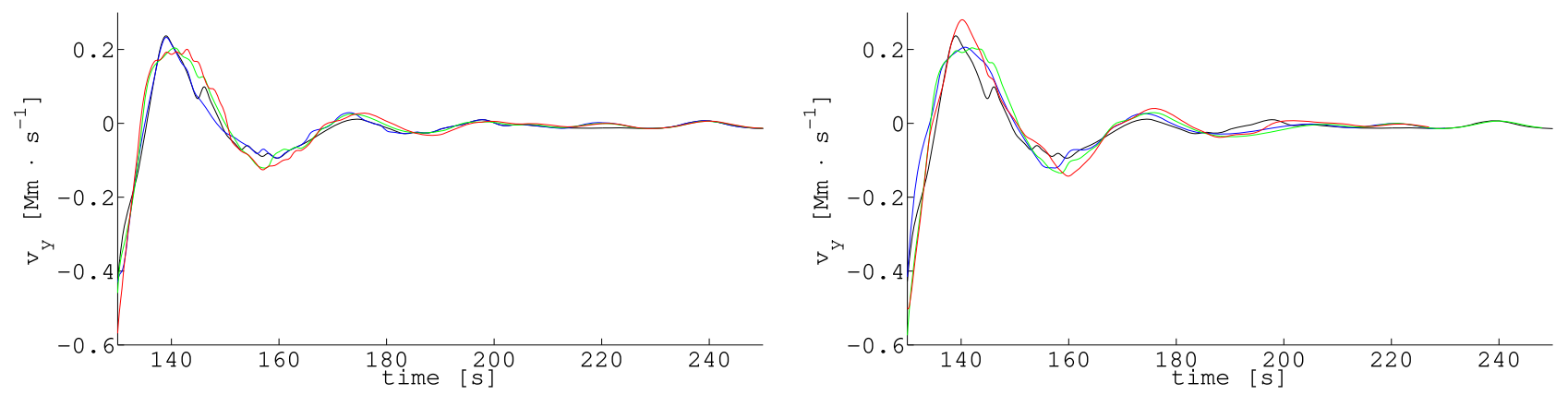

Figure 15. Temporal evolution of the $y$ component of the velocity $-v_{y}$ at the selected magnetic field line with the vector potential $A=-3.8$, showing the standing waves created after the interaction of the plasmoid with the magnetic arcade. In the left-hand panel, black, blue, green, and red lines correspond to the detection positions $x=0.0 ;-0.3125 ;-0.625$ and $-0.9375 \mathrm{Mm}$. In the right-hand panel, black, blue, green, and red lines correspond to the positions $x=0.0$; +0.3125 ; +0.625 and $+0.9375 \mathrm{Mm}$. The $y$ coordinates are placed along the selected magnetic field line.

observed in our model. Thus, the plasmoids may form the missing link for energy transport in flares. The plasmoids could be the agent transporting a large part of the energy from the flare site to the arcade. From there, the waves in the arcade may take over the energy transport. After coalescence with the underlying magnetic arcade, the plasmoid energy is now stored and transported in the arcade in the form of fast or Alfvén waves, following the scenario outlined by Fletcher \& Hudson (2008). This energy is then dissipated in the lower atmospheric layers by non-ideal effects (see, e.g., Reep \& Russell 2016). Such a scenario is a hybrid between previously proposed energy transport mechanisms in flares.

\section{Conclusions}

We numerically studied the oscillatory and wave processes in a gravitationally stratified current sheet, together with a realistic solar atmosphere structured as the VAL-C model, using the 2D time-dependent non-ideal (resistive) MHD equations solved by the FLASH numerical code, which implements AMR.

We summarize our results as follows. We generated an initial Gaussian pulse with the horizontal component of the velocity in the current sheet, which triggers the primary reconnection. It subsequently leads to the formation of a number of plasmoids. These plasmoids, under the gravitational and buoyancy forces start to move upward or downward. After some time, the formed plasmoids collide with each other as well as with the magnetic arcade formed in the lower altitudes of the solar atmosphere. We found that the magnetic arcade shrinks in the very early phases of evolution, in agreement with observations of downward motion of the X-ray source at the beginning of the 2003 November 3 flare (Veronig et al. 2006).

As a consequence of the plasmoid collisions, they very easily form much bigger plasmoids that oscillate. We observed in our simulation such a plasmoid with an oscillation period of $\approx 25 \mathrm{~s}$. This period is compatible with the calculated Alfvén transit time within the plasmoid. In line with the simulations of Kliem et al. (2000), Karlický \& Bárta (2007), and Karlický et al. (2010a), we expect that this oscillation process can generate the DPS with a "wavy" appearance on the radio spectrum (Karlický et al. 2016).

Moreover, for the first time, we have shown that plasmoids impacting a magnetic arcade can efficiently generate standing transverse waves of the slow magnetoacoustic type. The vertically polarized transverse waves have a period of $35 \mathrm{~s}$,

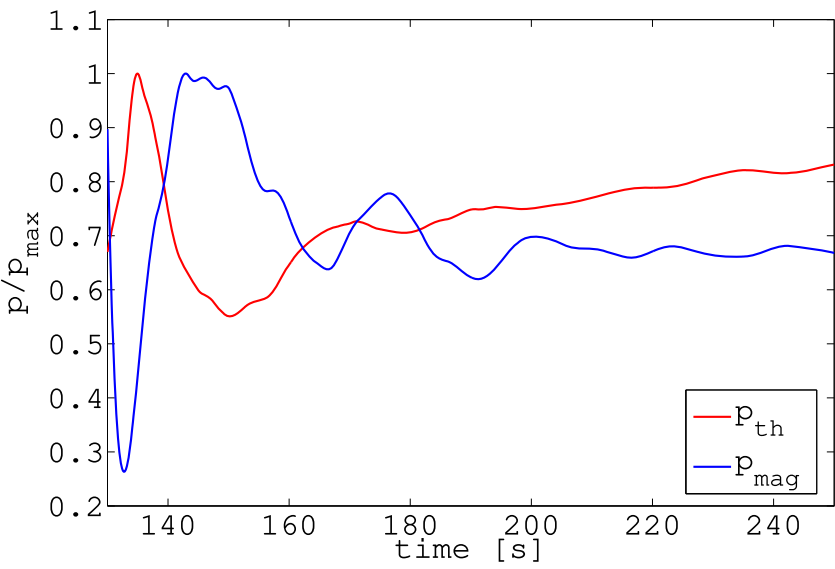

Figure 16. Temporal evolution of magnetic $p_{\text {mag }}$ and thermal $p_{\text {th }}$ pressure at the selected magnetic field line with the vector potential $A=-3.8$. Both pressures are in anti-phase, showing a presence of slow magnetoacoustic wave in the arcade. The detection point is placed at $x=+0.9375 \mathrm{Mm}$ and $y=4.1 \mathrm{Mm}$.

which is once again compatible with the Alfvén transit time in the arcade. The excitation of these transverse waves could be very important for explaining QPPs and energy propagation in a solar flare. We propose that plasmoids may carry away part of the energy from the reconnection site, which is consequently efficiently deposited in the underlying arcade, leading to wave dissipation in the (non-ideal) lower layers.

In our simulations, we neglected radiative and conductive losses. Although it is a drawback of the used model, oscillations of the plasmoid and arcade found in the present computations are very similar to those observed. For example, see Figures 5 and 6 in Karlický \& Kliem (2010), where the plasmoid and its oscillation in the brightness temperature and area are shown. The period of this oscillation was about $40 \mathrm{~s}$. On the other hand, in Figure 6(c) in Veronig et al. (2006), it can be seen that the velocity of the X-ray loop-top source shows a damped oscillation at the very beginning of the flare. Thus, these observations can be considered as supporting our results.

The oscillations of the arcade and plasmoid were strongly damped. When analyzing this process, we found that the oscillating plasmoid, as well as the oscillating arcade, are not isolated oscillating systems; both are strongly coupled with processes in the vertical current sheet through plasma flows. The downward oriented plasma flow, carrying plasmoids from 

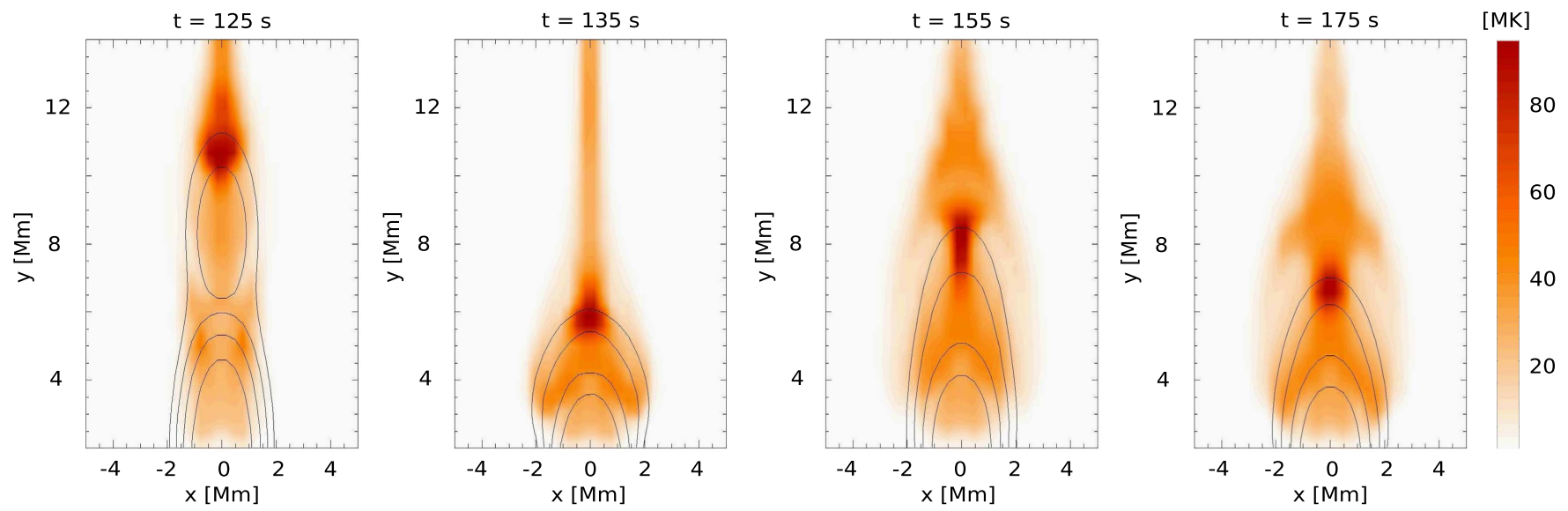

Figure 17. Time evolution (for $t=125,135,155,175 \mathrm{~s}$ ) of the temperature in the region of the coalescence of the plasmoid with the magnetic arcade.

the reconnection toward the arcade, not only triggers the arcade oscillations, but the flow damps this arcade oscillation subsequently. Similarly, the plasma flows in the vicinity of the oscillating plasmoid damp its oscillation.

We found that an interaction of the plasmoid with the flare arcade and merging of plasmoids generate the fast magnetoacoustic waves propagating out of the interaction place.

Finally, we found that in both processes (the merging of two plasmoids and interaction of a plasmoid with a flare arcade) the internal structure of the plasmoids as well as the arcades is very complex. Even an oscillating region with a very hot plasma close to the arcade top was generated. However, for a more realistic description of these hot plasma regions, the radiative and conductive losses need to be included into simulations, which constitutes future work. Furthermore, we will additionally add numerical resolution in the arcade regions, to allow for a better characterization of the wave nature.

The authors thank the referee for constructive comments that improved the paper. P.J., M.B. and M.K. acknowledge support from grants 16-13277S and 17-16447S of the Grant Agency of the Czech Republic. T.V.D. was supported by an Odysseus grant of the FWO Vlaanderen, the IAP P7/08 CHARM (Belspo), and the GOA-2015-014 (KU Leuven). The results were inspired by discussions at the ISSI Bern and at ISSI Beijing. The authors also express their thanks to Professor Kris Murawski for valuable discussions.

The FLASH code used in this work was developed by the DOE-supported ASC/Alliances Center for Astrophysical Thermonuclear Flashes at the University of Chicago.

\section{ORCID iDs}

P. Jelínek (1) https://orcid.org/0000-0002-7208-8342

T. Van Doorsselaere (10) https://orcid.org/0000-0001$9628-4113$

\section{References}

Aschwanden, M. J. 1987, SoPh, 111, 113

Aschwanden, M. J. 2005, Physics of the Solar Corona. An Introduction with Problems and Solutions (2nd ed.; Berlin: Springer)

Avrett, E. H., \& Loeser, R. 2008, ApJS, 175, 229

Bárta, M., Büchner, J., Karlický, M., \& Skála, J. 2011, ApJ, 737, 24

Bárta, M., Vršnak, B., \& Karlický, M. 2008, A\&A, 477, 649

Chen, S.-X., Li, B., Xiong, M., Yu, H., \& Guo, M.-Z. 2015, ApJ, 812, 22

Chen, S.-X., Li, B., Xiong, M., Yu, H., \& Guo, M.-Z. 2016, ApJ, 833, 114
Chung, T. J. 2002, Computational Fluid Dynamics (Cambridge: Cambridge Univ. Press)

De Moortel, I., \& Nakariakov, V. M. 2012, RSPTA, 370, 3193

Fang, X., Yuan, D., Xia, C., Van Doorsselaere, T., \& Keppens, R. 2016, ApJ, 833,36

Farge, M. 1992, AnRFM, 24, 395

Fárník, F., Karlický, M., \& Švestka, Z. 2003, SoPh, 218, 183

Fletcher, L., \& Hudson, H. S. 2008, ApJ, 675, 1645

Frost, K. J. 1969, ApJL, 158, L159

Fryxell, B., Olson, K., Ricker, P., et al. 2000, ApJS, 131, 273

Goedbloed, J. P. H., \& Poedts, S. 2004, Principles of Magnetohydrodynamics (Cambridge: Cambridge Univ. Press)

Guo, M.-Z., Chen, S.-X., Li, B., Xia, L.-D., \& Yu, H. 2016, SoPh, 291, 877

Huang, J., Tan, B., Zhang, Y., Karlický, M., \& Mészárosová, H. 2014, ApJ, 791, 44

Innocenti, M. E., Goldman, M., Newman, D., Markidis, S., \& Lapenta, G. 2015, ApJL, 810, L19

Jelínek, P., \& Karlický, M. 2012, A\&A, 537, A46

Jelínek, P., Karlický, M., \& Murawski, K. 2012, A\&A, 546, A49

Jelínek, P., Karlický, M., \& Murawski, K. 2015a, ApJ, 812, 105

Jelínek, P., Srivastava, A. K., Murawski, K., Kayshap, P., \& Dwivedi, B. N. 2015b, A\&A, 581, A131

Jiřička, K., \& Karlický, M. 2008, SoPh, 253, 95

Karlický, M., \& Bárta, M. 2007, A\&A, 464, 735

Karlický, M., \& Bárta, M. 2011, ApJ, 733, 107

Karlický, M., Bárta, M., \& Rybák, J. 2010a, A\&A, 514, A28

Karlický, M., Fárník, F., \& Krucker, S. 2004, A\&A, 419, 365

Karlický, M., Jiřička, K., \& Bárta, M. 2016, CEAB, 40, 93

Karlický, M., \& Kliem, B. 2010, SoPh, 266, 71

Karlický, M., Zlobec, P., \& Mészárosová, H. 2010b, SoPh, 261, 281

Kliem, B., Karlický, M., \& Benz, A. O. 2000, A\&A, 360, 715

Kołomański, S., \& Karlický, M. 2007, A\&A, 475, 685

Kolotkov, D. Y., Nakariakov, V. M., Kupriyanova, E. G., Ratcliffe, H., \& Shibasaki, K. 2015, A\&A, 574, A53

Kupriyanova, E. G., Melnikov, V. F., Nakariakov, V. M., \& Shibasaki, K. 2010, SoPh, 267, 329

Kupriyanova, E. G., Melnikov, V. F., \& Shibasaki, K. 2013, SoPh, 284, 559 Lee, D. 2013, JCoPh, 243, 269

Lee, D., \& Deane, A. E. 2009, JCoPh, 228, 952

Li, B., \& Li, X. 2008, ApJ, 682, 667

Li, B., Li, X., Hu, Y.-Q., \& Habbal, S. R. 2004, JGRA, 109, A07103

Mészárosová, H., Karlický, M., Rybák, J., Fárník, F., \& Jiřička, K. 2006 A\&A, 460, 865

Nakariakov, V. M., Arber, T. D., Ault, C. E., et al. 2004, MNRAS, 349, 705

Nakariakov, V. M., Foullon, C., Verwichte, E., \& Young, N. P. 2006, A\&A, 452,343

Nakariakov, V. M., Inglis, A. R., Zimovets, I. V., et al. 2010, PPCF, 52, 124009

Nakariakov, V. M., \& Melnikov, V. F. 2009, SSRv, 149, 119

Nakariakov, V. M., Melnikov, V. F., \& Reznikova, V. E. 2003, A\&A, 412, L7

Nakariakov, V. M., Pascoe, D. J., \& Arber, T. D. 2005, SSRv, 121, 115

Nakariakov, V. M., Pilipenko, V., Heilig, B., et al. 2016, SSRv, 200, 75

Nakariakov, V. M., \& Verwichte, E. 2005, LRSP, 2, 3

Nisticò, G., Pascoe, D. J., \& Nakariakov, V. M. 2014, A\&A, 569, A12

Norman, C. A., \& Smith, R. A. 1978, A\&A, 68, 145

Ohyama, M., \& Shibata, K. 1998, ApJ, 499, 934 
Parks, G. K., \& Winckler, J. R. 1969, ApJL, 155, L117

Pascoe, D. J. 2014, RAA, 14, 805

Priest, E. R. 1982, Solar Magneto-Hydrodynamics (Holland: D. Reidel), 195

Reep, J. W., \& Russell, A. J. B. 2016, ApJL, 818, L20

Roberts, B., Edwin, P. M., \& Benz, A. O. 1984, ApJ, 279, 857

Roberts, P. H. (ed.) 1967, An Introduction to Magnetohydrodynamics (London: Elsevier)

Russell, A. J. B., \& Fletcher, L. 2013, ApJ, 765, 81

Savage, S. L., Holman, G., Reeves, K. K., et al. 2012, ApJ, 754, 13

Solov'ev, A. A. 2010, ARep, 54, 86

Stepanov, A. V., Zaitsev, V. V., \& Nakariakov, V. M. 2012, Coronal Seismology: Waves and Oscillations in Stellar Coronae Flare Plasma (1st ed.; Weinheim: Wiley-VCH)
Tajima, T., Sakai, J., Nakajima, H., et al. 1987, ApJ, 321, 1031

Takasao, S., Asai, A., Isobe, H., \& Shibata, K. 2012, ApJL, 745, L6

Takasao, S., Matsumoto, T., Nakamura, N., \& Shibata, K. 2015, ApJ, 805, 135

Takasao, S., \& Shibata, K. 2016, ApJ, 823, 150

Tan, B. 2008, SoPh, 253, 117

Toro, E. F. 2006, IJNMF, 52, 433

Torrence, C., \& Compo, G. P. 1998, BAMS, 79, 61

Van Doorsselaere, T., Kupriyanova, E. G., \& Yuan, D. 2016, SoPh, 291, 3143

Vernazza, J. E., Avrett, E. H., \& Loeser, R. 1981, ApJS, 45, 635

Veronig, A. M., Karlický, M., Vršnak, B., et al. 2006, A\&A, 446, 675

Wang, T. J., Solanki, S. K., Innes, D. E., \& Curdt, W. 2005, A\&A, 435, 753

Yang, L., Zhang, L., He, J., et al. 2015, ApJ, 800, 111

Yu, H., Li, B., Chen, S.-X., Xiong, M., \& Guo, M.-Z. 2016, ApJ, 833, 51 